\title{
Guidelines \\ Current Indications of Secondary Enucleation in Retinoblastoma Management: A Position Paper on Behalf of the European Retinoblastoma Group (EURbG)
}

\author{
Christina Stathopoulos ${ }^{1, *}$, Livia Lumbroso-Le Rouic ${ }^{2}$, Annette C. Moll ${ }^{3}$, Manoj Parulekar ${ }^{4}$, Philippe Maeder ${ }^{5}$, \\ François Doz ${ }^{6}{ }^{\circledR}$, Helen Jenkinson ${ }^{7}$, Maja Beck Popovic ${ }^{8}$, Guillermo Chantada ${ }^{9} \mathbb{1}$ and Francis L. Munier ${ }^{1}$ \\ 1 Jules-Gonin Eye Hospital, Fondation Asile des Aveugles, University of Lausanne, \\ 1003 Lausanne, Switzerland; francis.munier@fa2.ch \\ 2 Department of Ocular Oncology, Institut Curie, 75005 Paris, France; livia.lumbroso@curie.fr \\ 3 Department of Ophthalmology, Amsterdam UMC, Vrije Universiteit Amsterdam, Cancer Center Amsterdam, \\ 1081 HV Amsterdam, The Netherlands; a.moll@amsterdamumc.nl \\ 4 Birmingham Children's Hospital Eye Department, Birmingham Women's and Children's NHS Foundation \\ Trust, Birmingham B4 6NH, UK; manoj.parulekar@nhs.net \\ 5 Department of Radiology, Centre Hospitalier Universitaire Vaudois, 1011 Lausanne, Switzerland; \\ philippe.maeder@chuv.ch \\ 6 SIREDO Centre (Care, Innovation and Research in Pediatric, Adolescent and Young Adult Oncology), \\ Institut Curie and University of Paris, 75005 Paris, France; francois.doz@curie.fr \\ check for \\ updates \\ Citation: Stathopoulos, C.; \\ Lumbroso-Le Rouic, L.; Moll, A.C.; \\ Parulekar, M.; Maeder, P.; Doz, F.; \\ Jenkinson, H.; Beck Popovic, M.; \\ 7 Department of Pediatric Oncology, Birmingham Women's and Children's NHS Foundation Trust, \\ Birmingham B4 6NH, UK; hjenkinson1@nhs.net \\ 8 Pediatric Hematology-Oncology Unit, Centre Hospitalier Universitaire Vaudois, 1010 Lausanne, Switzerland; \\ Maja.Beck-Popovic@chuv.ch \\ 9 Hospital Sant Joan de Deu, 08950 Barcelona, Spain; gchantada@yahoo.com \\ * Correspondence: christina.stathopoulos@fa2.ch
} Chantada, G.; Munier, F.L. Current Indications of Secondary Enucleation in Retinoblastoma Management: A Position Paper on Behalf of the European Retinoblastoma Group (EURbG). Cancers 2021, 13, 3392. https://doi.org/10.3390/cancers 13143392

Academic Editor: Salvatore Grisanti

Received: 25 May 2021

Accepted: 2 July 2021

Published: 6 July 2021

Publisher's Note: MDPI stays neutral with regard to jurisdictional claims in published maps and institutional affiliations.

Copyright: () 2021 by the authors. Licensee MDPI, Basel, Switzerland. This article is an open access article distributed under the terms and conditions of the Creative Commons Attribution (CC BY) license (https:// creativecommons.org/licenses/by/ $4.0 /)$.
Simple Summary: Although secondary enucleation (SE) is the treatment of choice for retinoblastoma eyes that did not respond favorably to conservative therapies, clear criteria for its indication are, however, currently missing. In this position paper on behalf of the European Retinoblastoma Group (EURbG), we discuss the available literature on SE, including its influence on metastases rate and survival, and propose guidelines to assist decision-making to interrupt eye-preserving therapies depending on the availabilities of advanced diagnostic and therapeutic modalities. Absolute indications to SE may be restricted to eyes with refractory tumor activity resisting all salvage treatments or eyes under apparent tumor control but no visual potential and irreducible complications. In contrast, eyes with an obscured optic nerve head and/or ocular complications amenable to specific surgical or medical management can be considered relative indications, provided that appropriate follow-up can be implemented and that parents are fully aware of a residual risk.

Abstract: Secondary enucleation (SE) puts an irreversible end to eye-preserving therapies, whenever their prolongation is expected to violate the presumed state of metastatic grace. At present, it must be acknowledged that clear criteria for SE are missing, leading to empiric and subjective indications commonly related to disease progression or relapse, disease persistence masking the optic nerve head or treatment-related complications obscuring the fundus view. This absence of evidence-based consensus regarding SE is explained by the continuously moving frontiers of the conservative management as a result of diagnostic and therapeutic advances, as well as by the lack of studies sufficiently powered to accurately stratify the risk of metastasis in conservatively treated patients. In this position paper of the European Retinoblastoma Group (EURbG), we give an overview of the progressive shift in the indications for SE over the past decades and propose guidelines to assist decision-making with respect to when SE becomes imperative or recommended, with corresponding absolute and relative SE indications. Further studies and validation of biologic markers correlated with the risk of metastasis are expected to set more precisely the frontiers of conservative management and thus consensual criteria for SE in the future. 
Keywords: retinoblastoma; secondary enucleation; metastasis; external beam irradiation; intravenous chemotherapy; intra-arterial chemotherapy; survival

\section{Introduction}

Despite the significant advances made in retinoblastoma management over the last decades, secondary enucleation (SE) is sometimes inevitable in order to preserve the patient from metastatic disease and death, and remains to date the treatment of choice for eyes that did not respond favorably to conservative strategies. Current indications to discontinue eye-preserving therapies include progressive/relapsing disease [1-42], persistent disease obscuring the optic nerve head, loss of fundus view (secondary to poor pupillary dilatation, intraocular hemorrhage and/or cataract) $[2,5,17,18,40,41,43,44]$, neovascular complications $[13,35,43,45]$, rhegmatogenous/tractional retinal detachment $[29,40]$, painful blind eye [16] and/or phthisis bulbi $[10,11,23]$. Sometimes, although rarely, SE may also be preferred by the parents due to the burden of prolonged conservative treatment on the child and/or family $[12,39]$, or encouraged by the medical team due to lack of parental compliance even if reasonable conservative alternatives can still be considered.

Although rarely performed for retinoblastoma groups A-C, various studies report SE rates for advanced retinoblastoma groups D-E ranging from $29 \%$ to $74 \%$ when treated with systemic chemotherapy $[18,22,46-48]$ and 0 to $61 \%$ when treated with intra-arterial chemotherapy $[23,30,34,38,49,50]$. Contrary to primary enucleation, where the presence of one or more International Intraocular Retinoblastoma Classification (IIRC) group E features at diagnosis such as neovascular glaucoma, massive intravitreal hemorrhage or diffuse infiltrating tumor have been considered, at least until recently, an absolute indication for immediate enucleation [51], clear consensual criteria for SE have never been established. In the event of disease progression and/or complications, the crucial decision to stop conservative management is therefore left to the expertise of the multidisciplinary team in charge, with the need to balance the potential benefits of additional globe-salvage treatments against the risks of disease progression and metastasis as well as to ensure full parental understanding of the potential consequences of retaining an eye.

The need to establish criteria for SE indications in the retinoblastoma management in order to harmonize the management and improve the care of retinoblastoma patients was raised by the European Retinoblastoma Group [52], a reference network for retinoblastoma involving more than 80 international experts of 24 countries dealing with retinoblastoma, in the annual meeting of 2019. A group of experts specialized in ocular oncology, pediatric oncology and neuroradiology from five major European Centers (Switzerland, France, England, Spain and Holland) were identified by the committee to address this question and provide guidelines for SE criteria.

\section{Methods}

Medline, Pubmed and Google Scholar were searched for English language scientific literature reporting on SE in retinoblastoma to summarize the actual knowledge on the topic. All studies with a minimum of 15 retinoblastoma patients managed conservatively and reporting on SE from January 1970 to December 2020 as well studies reporting exclusively on SE in retinoblastoma were included. Studies were categorized into three distinct groups: patients with SE after external beam irradiation $+/-$ focal therapies (Table 1), patients with SE after first line systemic chemotherapy $+/-$ focal therapies (Table 2) and patients with SE after first line or salvage intraarterial chemotherapy $+/-$ focal therapies (Table 3). Secondary enucleation rates and indications, clinico-pathological correlations of the enucleated eyes, adjuvant treatments, metastasis rates and overall survival if available were noted for each study. Series with fewer than 15 patients, case reports, non-English studies and studies on retinoblastoma management not reporting on SEs were excluded. When institutions published studies with similar cohorts, the ones with the larger ones 
and/or the ones with the more detailed information including longer follow-ups were chosen. Independent screening of the literature using the inclusion/exclusion criteria was performed by two of the authors (C.S., F.L.M.).

Based on the results of the above-described literature review, we first discuss indications and metastasis rate in line with the different eras of disease management and finally propose SE criteria and follow-up guidelines for characteristic clinical situations as a result of a consensus made on behalf of the European Retinoblastoma Group (EURbG) by 10 retinoblastoma experts in ocular oncology (C.S., L.L.-L.R., A.M., M.P., F.L.M.) pediatric oncology (F.D., H.J., G.C., M.B.P.) and neuroradiology (P.M.). 
Table 1. Secondary enucleations (SE), orbital invasion, metastases and mortality rates after first-line external beam radiation therapy (EBRT): literature review.

\begin{tabular}{|c|c|c|c|c|c|c|c|c|c|c|c|c|c|c|c|}
\hline \multirow{2}{*}{$\begin{array}{c}\text { Author } \\
\text { (Year) }\end{array}$} & \multirow{2}{*}{ Study Years } & \multicolumn{2}{|c|}{ Eyes/Patients } & \multicolumn{2}{|c|}{ Classification Group } & \multicolumn{2}{|c|}{ Treatments † } & \multirow{2}{*}{$\begin{array}{c}\text { Indication for SE } n \\
(\% \text { SE Eyes) }\end{array}$} & \multirow{2}{*}{$\begin{array}{l}\text { Mean/Median } \\
\text { Retention Time, } \\
\text { Months (Range) }\end{array}$} & \multirow{2}{*}{$\begin{array}{c}\text { HRF, } n \text { (\%) SE } \\
\text { Eyes) }\end{array}$} & \multirow{2}{*}{$\begin{array}{l}\text { Adjuvant IVC, } \\
n \text { (\%) SE Eyes } \\
\text { with HRFF }\end{array}$} & \multirow{2}{*}{$\begin{array}{c}\text { Mean/Median } \\
\text { Follow-Up, } \\
\text { Months (Range) }\end{array}$} & \multirow{2}{*}{$\begin{array}{c}\text { Orbit } \\
\text { Invasion, } \\
(\% \text { Patients) }\end{array}$} & \multirow{2}{*}{$\begin{array}{l}\text { Metastases, } n \\
\text { (\% Patients) }\end{array}$} & \multirow{2}{*}{$\begin{array}{c}\text { Deaths, } n \text { (\%) } \\
\text { Patients) }\end{array}$} \\
\hline & & All & SE Eyes & All & SE Eyes & All & SE Eyes $^{++}$ & & & & & & & & \\
\hline $\begin{array}{l}\text { Thompson et al. } \\
(1972)[1]\end{array}$ & 1956-1970 & $34 / 33$ & 9/9 & $\begin{array}{c}\text { RE: } \\
\mathrm{I}=17 \\
\mathrm{II}=3 \\
\mathrm{III}=5 \\
\mathrm{IV}=4 \\
\mathrm{~V}=5 \\
\end{array}$ & $\begin{array}{c}\text { RE: } \\
I=1 \\
I I=2 \\
\text { III }=2 \\
\text { V }=2 \\
V=2\end{array}$ & $\mathrm{EBRT}=34$ & EBRT $=9$ & Active disease $=9(100)^{*}$ & $17(4-43)$ & na & nа & na & nа & $1(3)^{\mathrm{a}}$ & $1(3)^{\mathrm{a}}$ \\
\hline $\begin{array}{l}\text { Egbert te al. } \\
(1978)[2]\end{array}$ & 1956-1974 & 38 & 16 & $\begin{array}{l}\text { RE: } \\
I=8 \\
\text { II }=5 \\
\text { III }=8 \\
\text { IV }=2 \\
\mathrm{~V}=15\end{array}$ & $\begin{array}{l}\text { RE: } \\
\mathrm{I}=1 \\
\mathrm{II}=2 \\
\mathrm{III}=1 \\
\mathrm{IV}=2 \\
\mathrm{~V}=10\end{array}$ & EBRT $=38$ & EBRT $=16$ & $\begin{array}{c}\text { Active disease }=7(44) * \\
\text { Blind eye }=8(50) \\
-2 \text { ry NVG }=5 \\
-2 \mathrm{ry} \mathrm{VH}=1 \\
-2 \mathrm{Vr}=1 \\
-2 \mathrm{ry} \text { cataract }=1 \\
\quad \text { Unknown }=1(6)\end{array}$ & $(0-4$ years $)$ & na & na & $\begin{array}{c}10 \text { years } \\
(2.5-21 \text { years })\end{array}$ & na & na & $0(0)$ \\
\hline $\begin{array}{l}\text { Foote et al. } \\
\text { (1989) [3] }\end{array}$ & 1977-1987 & $25 / 18$ & $5 /$ na & $\begin{array}{c}\text { RE: } \\
I=1 \\
I I=1 \\
I I I=13 \\
I V=4 \\
V=6\end{array}$ & $\begin{array}{c}\text { RE: } \\
\text { III }=2 \\
\mathrm{IV}=2 \\
\mathrm{~V}=1\end{array}$ & EBRT $=25$ & EBRT $=5$ & Active disease $=5(100)^{*}$ & $10(2-25)$ & na & na & $32(7-113)$ & na & nа & $0(0)$ \\
\hline $\begin{array}{l}\text { Toma et al. } \\
\text { (1995) }[4]\end{array}$ & 1986-1992 & $67 / 53$ & $5 /$ na & $\begin{array}{l}\text { RE: } \\
\mathrm{I}=18 \\
\mathrm{II}=33 \\
\mathrm{III}=11 \\
\mathrm{IV}=5\end{array}$ & $\begin{array}{c}\text { RE: } \\
\mathrm{II}=4 \mathrm{III}=1\end{array}$ & $\mathrm{EBRT}=67$ & EBRT $=5$ & Active disease $=5(100)^{*}$ & na & na & na & $35(12-82)$ & na & na & na \\
\hline $\begin{array}{l}\text { Hernandez et al. } \\
\quad(1996)[5]\end{array}$ & 1980-1991 & $34 / 27$ & $9 /$ na & $\begin{array}{c}\text { RE: } \\
\text { I-II }=14 \\
\text { III }=7 \\
\text { IV-V }=13\end{array}$ & nа & $\begin{array}{c}E B R T=27 \\
\text { Plaque }=\text { some }\end{array}$ & $\begin{array}{c}E B R T=9 \\
\text { Plaque }=2\end{array}$ & $\begin{array}{l}\text { Active disease }=7(67)^{*} \\
\text { Irradiation complications } \\
=2(33) \\
- \text { Glaucoma }=1 \\
-\mathrm{NVX}+\text { hemorrhage }=1\end{array}$ & na & na & na & $35(12-93)$ & $0(0)$ & $0(0)$ & $0(0)$ \\
\hline $\begin{array}{l}\text { Blach et al. } \\
\text { (1996) [6] }\end{array}$ & 1979-1991 & $180 / 123$ & $32 / \mathrm{na}$ & $\begin{array}{c}\text { RE: } \\
I=41 \\
I I=32 \\
I I I=22 \\
I V=13 \\
V=72\end{array}$ & $\begin{array}{c}\mathrm{RE}: \\
\mathrm{I}-\mathrm{III}=8 \\
\mathrm{IV} / \mathrm{V}=24\end{array}$ & EBRT $=182$ & $\mathrm{EBRT}=32$ & Active disease $=32(100)^{*}$ & na & na & $27(84)^{\mathrm{b}}$ & $(4-159)$ & na & $6(5)^{c}$ & $14(11)^{\mathrm{c}}$ \\
\hline $\begin{array}{l}\text { Merchant et al. } \\
\text { ocular (2002) [7] }\end{array}$ & 1978-1998 & $49 / 38$ & $6 /$ na & $\begin{array}{l}\text { RE: } \\
\mathrm{I}=14 \\
\mathrm{II}=10 \\
\mathrm{III}=11 \\
\mathrm{IV}=2 \\
\mathrm{~V}=7 \\
\text { na }=5\end{array}$ & na & $\begin{array}{l}\mathrm{EBRT}=49 \\
\operatorname{IVC}=10\end{array}$ & EBRT $=6$ & Active disease $=6(100)^{*}$ & $15(7-68)$ & na & nа & $89(34-373)$ & $1(3)^{\mathrm{d}}$ & $0(0)$ & $4(11)^{d}$ \\
\hline $\begin{array}{l}\text { Phillips et al. } \\
\text { (2003) [8] }\end{array}$ & 1965-1997 & $47 / 35$ & 13 & $\begin{array}{c}\text { RE: } \\
\mathrm{IIII}=16 \\
\mathrm{III}=7 \\
\mathrm{IV}-\mathrm{V}=20 \\
\mathrm{na}=4\end{array}$ & $\begin{array}{c}\mathrm{RE}: \\
\mathrm{IIII}=1 \\
\mathrm{III}=1 \\
\mathrm{IV}=1 \\
\mathrm{~V}=8 \\
\mathrm{na}=2\end{array}$ & $\begin{array}{l}\mathrm{EBRT}=47 \\
\mathrm{IVC}=9 \mathrm{e}\end{array}$ & $\mathrm{EBRT}=13$ & na & $\begin{array}{l}\text { within } 2 \text { years } \\
\text { post EBRT }\end{array}$ & na & na & na & na & $1(3)^{\mathrm{f}}$ & $5(14)^{\mathrm{f}}$ \\
\hline $\begin{array}{l}\text { Abramson et al. } \\
(2004)[9]\end{array}$ & 1979-2002 & $63 / 53$ & $25 / \mathrm{na}$ & $\begin{array}{c}\text { RE: } \\
\mathrm{Vb}=63\end{array}$ & $\begin{array}{c}\text { RE: } \\
\mathrm{Vb}=25\end{array}$ & EBRT $=63$ & EBRT $=25$ & Active disease $=25(100)^{*}$ & $14(5-107)$ & na & na & na & $2(4)^{g}$ & $3(6)$ & $5(9)^{\mathrm{h}}$ \\
\hline
\end{tabular}


Table 1. Cont.

\begin{tabular}{|c|c|c|c|c|c|c|c|c|c|c|c|c|c|c|c|}
\hline \multirow{2}{*}{$\begin{array}{l}\text { Author } \\
\text { (Year) }\end{array}$} & \multirow{2}{*}{ Study Years } & \multicolumn{2}{|c|}{ Eyes/Patients } & \multicolumn{2}{|c|}{ Classification Group } & \multicolumn{2}{|c|}{ Treatments +} & \multirow{2}{*}{$\begin{array}{l}\text { Indication for SE } n \\
(\% \text { SE Eyes })\end{array}$} & \multirow{2}{*}{$\begin{array}{l}\text { Mean/Median } \\
\text { Retention Time, } \\
\text { Months (Range) }\end{array}$} & \multirow{2}{*}{$\begin{array}{c}\text { HRF, } n \\
(\% \text { SE Eyes) }\end{array}$} & \multirow{2}{*}{$\begin{array}{l}\text { Adjuvant IVC, } \\
n \text { (\%) SEeses } \\
\text { with HRF) }\end{array}$} & \multirow{2}{*}{$\begin{array}{l}\text { Mean/Median } \\
\text { Follow-U, } \\
\text { Months (Range) }\end{array}$} & \multirow{2}{*}{$\begin{array}{c}\text { Orbit } \\
\text { Invasion, } n \\
(\% \text { Patients) }\end{array}$} & \multirow{2}{*}{$\begin{array}{l}\text { Metastases, } n \\
\text { (\% Patients) }\end{array}$} & \multirow{2}{*}{$\begin{array}{l}\text { Deaths, } n \text { (\%) } \\
\text { Patients) }\end{array}$} \\
\hline & & All & SE Eyes & All & SE Eyes & All & SE Eyes ${ }^{++}$ & & & & & & & & \\
\hline $\begin{array}{l}\text { Choi et al. } \\
\text { (2010) }[10]\end{array}$ & 1987-1998 & $32 / 25$ & $8 / 7$ & $\begin{array}{l}\text { RE: } \\
\text { III }=5 \\
\text { III }=16 \\
\text { IV }=4 \\
\text { V }\end{array}$ & $\begin{array}{l}\text { RE: } \\
\text { III }=1 \\
\text { III }=3 \\
\text { V }=2 \\
\text { = }\end{array}$ & $\begin{array}{l}\mathrm{EBRT}=32 \\
\mathrm{IVC}=22^{\mathrm{i}}\end{array}$ & EBRT $=8$ & $\begin{array}{c}\text { Active disease }=7(88)^{*} \\
\text { Cataract + phthisis }=1 \text { (12) }\end{array}$ & na & na & па & $150(55-249)$ & na & $2(8)$ & $2(8)^{j}$ \\
\hline $\begin{array}{l}\text { Camp et al. } \\
\text { (2019) [11] }\end{array}$ & 1980-1994 & $48 / 26$ & 4/na & na & na & $\begin{array}{c}\text { EBRT }=24 \\
\text { Plaque } / \mathrm{FT}=24\end{array}$ & EBRT era ${ }^{k}$ & $\begin{array}{c}\text { Active disease }=2(50) * \\
\text { NVG }=1(25) \\
\text { Phthisis }=1(25)\end{array}$ & na & na & nа & $125(4-330)$ & na & 1(4) ${ }^{1}$ & $5(19)^{1}$ \\
\hline
\end{tabular}

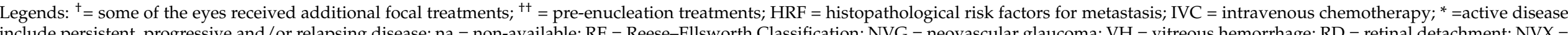

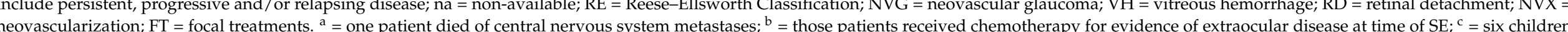

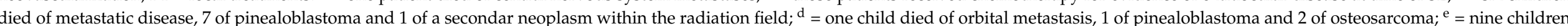

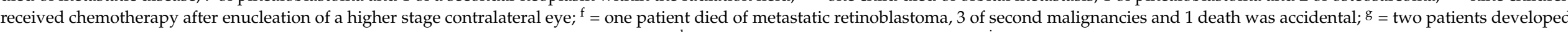

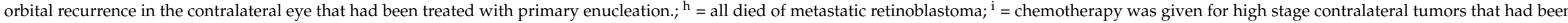

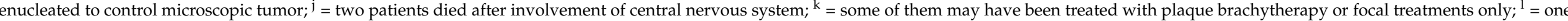
patient died of metastasis, 2 of second malignancies, 1 of pinealoblastoma and 1 of respiratory failure.

Table 2. Secondary enucleations (SE), orbital invasion, metastases and mortality rates after first-line intravenous chemotherapy (IVC): literature review.

\begin{tabular}{|c|c|c|c|c|c|c|c|c|c|c|c|c|c|c|c|}
\hline \multirow{2}{*}{ Author (Year) } & \multirow{2}{*}{ Study Years } & \multicolumn{2}{|c|}{ Eyes/Patients } & \multicolumn{2}{|c|}{ Classification Group } & \multicolumn{2}{|c|}{ Treatments +} & \multirow{2}{*}{$\begin{array}{c}\text { Indication for SE } n \\
\text { (\% SE Eyes) }\end{array}$} & \multirow{2}{*}{$\begin{array}{l}\text { Mean/Median } \\
\text { Retention Time, } \\
\text { Months (Range) }\end{array}$} & \multirow{2}{*}{$\begin{array}{c}\mathrm{HRE}, n \\
\text { (\% SE Eyes) }\end{array}$} & \multirow{2}{*}{$\begin{array}{l}\text { Adjuvant IVC, } \\
n(\% \text { ( } \% \text { Eyes } \\
\text { with HRF) }\end{array}$} & \multirow{2}{*}{$\begin{array}{l}\text { Mean/Median } \\
\text { Follow-Up, } \\
\text { Months (Range) }\end{array}$} & \multirow{2}{*}{$\begin{array}{l}\text { Orbit Invasion, } \\
n \text { (\% Patients) }\end{array}$} & \multirow{2}{*}{$\begin{array}{l}\text { Metastases, } n \\
\text { (\% Patients) }\end{array}$} & \multirow{2}{*}{$\begin{array}{c}\text { Deaths, } n \\
\text { (\% Patients) }\end{array}$} \\
\hline & & All & SE Eyes & All & SE Eyes & All & SE Eyes ${ }^{t+}$ & & & & & & & & \\
\hline $\begin{array}{l}\text { Nenadovo Beck } \\
\text { et al. (2000) [12] }\end{array}$ & 1995-1998 & $33 / 24$ & $6 / 5$ & $\begin{array}{c}\text { RE: } \\
I=5 \\
I I=10 \\
\text { III }=3 \\
I V=1 \\
V=14 \\
\end{array}$ & $\begin{array}{l}\text { RE: } \\
V=5\end{array}$ & $\begin{array}{l}\mathrm{IVC}=33 \\
\mathrm{EBRT}=13\end{array}$ & $\begin{array}{l}I V C=6 \\
\mathrm{EBRT}=2\end{array}$ & $\begin{array}{l}\text { Active disease }=5(83) \\
\text { Parental wish }=1(17)\end{array}$ & $(0-8)$ & na & na & $31(4-41)$ & $0(0)$ & $1(4)^{\mathrm{a}}$ & $1(4)^{\mathrm{a}}$ \\
\hline $\begin{array}{l}\text { Rodriguez- } \\
\text { Galindo et al. } \\
\text { (2003) [13] }\end{array}$ & $1996-2000$ & $43 / 25$ & $13 / 11$ & $\begin{array}{c}\text { RE: } \\
I=7 \\
\mathrm{II}=12 \\
\mathrm{III}=5 \\
\mathrm{IV}=3 \\
\mathrm{~V}=16\end{array}$ & $\begin{array}{c}\text { RE: } \\
\text { II }=2 \\
\text { III }=2 \\
\text { IV }=3 \\
V=6\end{array}$ & $\begin{array}{l}\text { IVC }=43 \\
\mathrm{EBRT}=19\end{array}$ & $\begin{array}{l}\text { IVC }=13 \\
\text { EBRT }=9\end{array}$ & $\begin{array}{c}\text { Active disease }=10(77) * \\
\text { NVG }=3(23)\end{array}$ & $(14 ; 8-33)$ & $3(23)^{b}$ & $3(100)$ & $32(10-65)$ & na & na & $0(0)$ \\
\hline $\begin{array}{l}\text { Schiavetti et al. } \\
\text { (2005) [15] }\end{array}$ & 1992-na & $58 / 46$ & $21 / \mathrm{na}$ & $\begin{array}{l}\text { RE: } \\
\mathrm{I}=10 \\
\mathrm{II}=16 \\
\mathrm{III}=9 \\
\mathrm{IV}=6 \\
\mathrm{~V}=17\end{array}$ & $\begin{array}{l}\text { RE: } \\
I=1 \\
I I=4 \\
\text { III }=3 \\
\text { IV }=2 \\
V=11\end{array}$ & $\begin{array}{l}\mathrm{IVC}=58 \\
\mathrm{EBRT}=10\end{array}$ & $\begin{array}{l}\mathrm{IVC}=21 \\
\mathrm{EBRT}=3\end{array}$ & Active disease $=21(100)^{*}$ & па & na & na & $53(11-125)$ & па & $1(2)^{\mathrm{d}}$ & $4(9)^{d}$ \\
\hline
\end{tabular}


Table 2. Cont.

\begin{tabular}{|c|c|c|c|c|c|c|c|c|c|c|c|c|c|c|c|}
\hline \multirow{2}{*}{ Author (Year) } & \multirow{2}{*}{ Study Years } & \multicolumn{2}{|c|}{ Eyes/Patients } & \multicolumn{2}{|c|}{ Classification Group } & \multicolumn{2}{|c|}{ Treatments +} & \multirow{2}{*}{$\begin{array}{c}\text { Indication for SE } n \\
\text { (\% SE Eyes) }\end{array}$} & \multirow{2}{*}{$\begin{array}{l}\text { Mean/Median } \\
\text { Retention Time, } \\
\text { Months (Range) }\end{array}$} & \multirow{2}{*}{$\begin{array}{c}\text { HRF, } n \\
\text { (\% SE Eyes) }\end{array}$} & \multirow{2}{*}{$\begin{array}{l}\text { Adjuvant IVC, } \\
n(\% \text { SE Eyes } \\
\text { with HRF) }\end{array}$} & \multirow{2}{*}{$\begin{array}{l}\text { Mean/Median } \\
\text { Follow-Up, } \\
\text { Months (Range) }\end{array}$} & \multirow{2}{*}{$\begin{array}{l}\text { Orbit Invasion, } \\
n \text { (\% Patients) }\end{array}$} & \multirow{2}{*}{$\begin{array}{l}\text { Metastases, } n \\
\text { (\% Patients) }\end{array}$} & \multirow{2}{*}{$\begin{array}{c}\text { Deaths, } n \\
\text { (\% Patients) }\end{array}$} \\
\hline & & All & SE Eyes & All & SE Eyes & All & SE Eyes $^{t+}$ & & & & & & & & \\
\hline $\begin{array}{l}\text { Chantada et al. } \\
(2007)[16]\end{array}$ & 1995-2002 & - & 139/122 & - & $\begin{array}{c}\text { RE: } \\
\mathrm{I}-\mathrm{IV}=25 \\
\mathrm{~V}=91 \\
\mathrm{na}=23\end{array}$ & - & $\begin{array}{l}\text { IVC }=139 \\
\text { EBRT }=35\end{array}$ & $\begin{array}{l}\text { Active disease }=136(98) * \\
\text { Painful blind eye }=3(2)^{*}\end{array}$ & $(10 ; 1-90)^{\mathrm{f}}$ & $41(29) \mathrm{g}$ & $8(20)^{\mathrm{h}}$ & $(54 ; 12-97)$ & $2(2)^{\mathrm{i}}$ & $3(2)^{i, j}$ & $2(2)^{\mathrm{i}, j}$ \\
\hline $\begin{array}{l}\text { Lumbroso-Le } \\
\text { Rouic et al. } \\
\text { (2008) [17] }\end{array}$ & 1998-2002 & $115 / 83$ & $23 / 20$ & $\begin{array}{l}\text { IIRC: } \\
\mathrm{A}=19 \\
\mathrm{~B}=48 \\
\mathrm{C}=19 \\
\mathrm{D}=29\end{array}$ & $\begin{array}{l}\text { IIRC: } \\
B=2 \\
C=4 \\
D=17\end{array}$ & $\begin{array}{l}\mathrm{IVC}=115 \\
\mathrm{EBRT}=13\end{array}$ & $\begin{array}{l}\mathrm{IVC}=23 \\
\mathrm{EBRT}=7\end{array}$ & $\begin{array}{l}\text { Active disease }=18(78)^{*} \\
\text { No fundus view }=5(22) \\
-2 \text { ry to massive } V H=5\end{array}$ & na & na & na & $51(32-72)$ & na & na & na \\
\hline $\begin{array}{l}\text { Shin et al. } \\
\text { (2010) [18] }\end{array}$ & 1997-2007 & $65 / 52$ & $31 / \mathrm{na}$ & $\begin{array}{l}\text { IIRC: } \\
\mathrm{A}=8 \\
\mathrm{~B}=14 \\
\mathrm{D}=42 \\
\mathrm{E}=1\end{array}$ & $\begin{array}{l}\text { IIRC: } \\
B=4 \\
D=27\end{array}$ & $\mathrm{IVC}=65$ & $\mathrm{IVC}=31$ & $\begin{array}{l}\text { Active disease }=29(94)^{*} \\
\text { No fundus view }=2(6) \\
-2 \text { ry hyphema and } \mathrm{VH}=1 \\
-2 \text { ry cataract }=1\end{array}$ & na & na & na & $54(7-115)$ & na & na & $1(2)^{\mathrm{k}}$ \\
\hline $\begin{array}{l}\text { Zhao et al. } \\
\text { (2011) [19] }\end{array}$ & 2006-2008 & - & $55 / 55$ & - & $\begin{array}{l}\text { IIRC: } \\
\mathrm{D}=10 \\
\mathrm{E}=45\end{array}$ & - & $\mathrm{IVC}=55$ & Active disease $=55(100)^{*}$ & $3(0.2-19)$ & $7(13)^{1}$ & some & $25(12-38)$ & na & $5(9)$ & $5(9) \mathrm{m}$ \\
\hline $\begin{array}{l}\text { Bartuma et al. } \\
(2014)[20]\end{array}$ & 2001-2011 & $46 / 24$ & $13 / 12$ & $\begin{array}{l}\text { IIRC: } \\
A=8 \\
B=25 \\
C=1 \\
D=11 \\
E=1\end{array}$ & $\begin{array}{l}\text { IIRC: } \\
B=3 \\
\mathrm{C}=1 \\
\mathrm{D}=7 \\
\mathrm{E}=1\end{array}$ & $\begin{array}{l}\mathrm{IVC}=46 \\
\mathrm{EBRT}=4\end{array}$ & $\begin{array}{l}\mathrm{IVC}=12 \\
\mathrm{EBRT}=3\end{array}$ & $\begin{array}{l}\text { Active disease }=12(100)^{*} \\
-\mathrm{VS} \text { and /or } S R S=5 \\
- \text { large retinal tumor }=3 \\
- \text { growth on the ON }=3 \\
\text { - large tumor and extensive } \\
\mathrm{RD}=2\end{array}$ & $14(1-43)$ & $3(25)^{\mathrm{n}}$ & $0(0)$ & $60(13-144)$ & $0(0)$ & $0(0)$ & $0(0)$ \\
\hline $\begin{array}{l}\text { Brennan et al. } \\
(2015)[21]\end{array}$ & 1997-2013 & - & $63 / 60$ & - & $\begin{array}{l}\text { IIRC: } \\
B=13 \\
C=7 \\
D=34 \\
E=3 \\
\text { na }=6\end{array}$ & - & $\begin{array}{l}\mathrm{IVC}=62 \\
\mathrm{IAC}=1 \\
\text { ERRT }=26 \\
\text { Plaque }=10\end{array}$ & $\begin{array}{c}\text { Active disease }=56(89)^{*} \\
\mathrm{VS}=40 \\
\mathrm{SRS}=21 \\
\mathrm{RD}=39 \\
\text { Other }=7(11)^{\circ}\end{array}$ & $\begin{array}{c}19 \text { (1-13 years) } \\
\text { With HRF: } \\
30 \text { (1-157) } \\
\text { With no HRF: } \\
16(1-72) \\
p=0.018\end{array}$ & $13(21) \mathrm{P}$ & $10(77) \mathrm{q}$ & $0(0)$ & $0(0)$ & $0(0)$ & $0(0)$ \\
\hline $\begin{array}{l}\text { Berry et al. } \\
\text { (2017) [22] }\end{array}$ & 1995-2015 & $139 /$ na & $58 /$ na & $\begin{array}{l}\text { IIRC: } \\
\mathrm{D}=102 \\
\mathrm{E}=37\end{array}$ & $\begin{array}{l}\text { IIRC: } \\
\mathrm{D}=30 \\
\mathrm{E}=28\end{array}$ & $\begin{array}{l}\mathrm{IVC}=139 \\
\mathrm{EBRT}=39\end{array}$ & $\begin{array}{c}\mathrm{IVC} \pm \mathrm{EBRT} \\
58\end{array}$ & Active disease $=58(100)^{*}$ & $14(8 ; 1-118)$ & $0(0)$ & $0(0)$ & 86 & 1(1) & $1(1)^{\mathrm{r}}$ & $1(1)^{\mathrm{r}}$ \\
\hline $\begin{array}{c}\text { Munier et al. } \\
(2017)[23]\end{array}$ & 1997-2008 & $23 / 23$ & $10 / 10$ & $\begin{array}{l}\text { IIRC: } \\
D=23\end{array}$ & $\begin{array}{l}\text { IIRC: } \\
D=10\end{array}$ & $\begin{array}{l}\text { IVC }=23 \\
\text { Plaque }=2\end{array}$ & $\operatorname{IVC} \pm$ plaque $=$ & $\begin{array}{c}\text { Active disease }=9(90)^{*} \\
\text { seeding } \pm \mathrm{RD}=9 \\
\text { Phthisis bulbi }=1(10)\end{array}$ & $17(1-48)$ & $2(20)$ & $2(100)$ & $105(29-218)$ & $0(0)$ & $0(0)$ & $0(0)$ \\
\hline $\begin{array}{l}\text { Fabian et al. } \\
\text { (2017) [24] }\end{array}$ & 2002-2014 & - & $24 /$ na & - & $\begin{array}{l}\text { IIRC: } \\
D=24\end{array}$ & - & $\begin{array}{c}\text { IVC }=24 \\
\mathrm{IAC}=11 \text { plaque } \\
=5 \mathrm{EBRT}=5 \\
\mathrm{IvitC}=4 \mathrm{~s}\end{array}$ & $\begin{array}{c}\text { Active disease }=5(21)^{*} \\
-\mathrm{VS}=2 \\
-\mathrm{AC}=2 \\
-\mathrm{V} \text { Vitreous base relapse }+ \\
\text { diffuse subretinal } \\
\text { hemorrhage }=1 \\
\text { na }=19(79)\end{array}$ & $\begin{array}{c}\text { With HRF: } \\
27(17-40)^{\mathrm{s}} \\
\text { Without HRF: } 9 \\
(3-91)^{\mathrm{s}}\end{array}$ & $5(21)^{t}$ & $5(100)$ & $72(14-153) \mathrm{u}^{\mathrm{u}}$ & $0(0)$ & $0(0)$ & $0(0)$ \\
\hline $\begin{array}{l}\text { Shields et al. } \\
\text { (2020) [25] }\end{array}$ & 1994-2019 & $964 / 554$ & $161 /$ na & $\begin{array}{l}\text { ICRB: } \\
\mathrm{A}=54 \\
\mathrm{~B}=5200 \\
\mathrm{C}=128 \\
\mathrm{D}=224 \\
\mathrm{E}=225 \\
\mathrm{na}=123\end{array}$ & $\begin{array}{l}\text { ICRB: } \\
\mathrm{A}=1 \\
\mathrm{~B}=9 \\
\mathrm{C}=4 \\
\mathrm{D}=41 \\
\mathrm{E}=82 \\
\mathrm{n}=24\end{array}$ & $\begin{array}{c}\mathrm{IVC} \pm \mathrm{IAC} \pm \\
\text { plaque } \pm \text { EBRT } \\
\pm \text { IvitC }=964\end{array}$ & $\begin{array}{c}\mathrm{IVC} \pm \mathrm{IAC} \pm \\
\text { plaque } \pm \text { EBRT } \\
\pm \text { Ivite }=161\end{array}$ & na & $15(10,1-191)$ & na & na & $77(0-299)^{v}$ & na & $11(2)$ & $7(1)^{\mathrm{w}}$ \\
\hline $\begin{array}{l}\text { Gündüz et al. } \\
\text { (2020) [26] }\end{array}$ & 1998-2018 & 276/na & $75 /$ na & $\begin{array}{l}\text { ICRB: } \\
\mathrm{A}=22 \\
\mathrm{~B}=114 \\
\mathrm{C}=28 \\
\mathrm{D}=90 \\
\mathrm{E}=97\end{array}$ & $\begin{array}{c}\text { ICRB: } \\
B=16 \\
C=4 \\
D=49 n a \\
E=81\end{array}$ & $\begin{array}{c}\text { IVC }=254 \\
\text { IAC }=3 \\
\text { FT }=19 \\
\pm \text { EBRT } \\
\pm \text { Plaque } \\
\pm \text { salvage IAC }\end{array}$ & $\begin{array}{c}\text { IVC } \pm \text { EBRT } \pm \\
\text { IAC } \\
\text { Plaque }=9\end{array}$ & Active disease $=75(100)^{*}$ & na & $10(13)$ & $10(100)$ & $77(1-268)$ & na & $1(\mathrm{na})^{x}$ & $2(\mathrm{na})^{x}$ \\
\hline
\end{tabular}


Table 2. Cont.

\begin{tabular}{|c|c|c|c|c|c|c|c|c|c|c|c|c|c|c|c|}
\hline \multirow{2}{*}{ Author (Year) } & \multirow{2}{*}{ Study Years } & \multicolumn{2}{|c|}{ Eyes/Patients } & \multicolumn{2}{|c|}{ Classification Group } & \multicolumn{2}{|c|}{ Treatments $\dagger$} & \multirow{2}{*}{$\begin{array}{c}\text { Indication for SE } n \\
\text { (\% SE Eyes) }\end{array}$} & \multirow{2}{*}{$\begin{array}{l}\text { Mean/Median } \\
\text { Retention Time, } \\
\text { Months (Range) }\end{array}$} & \multirow{2}{*}{$\begin{array}{l}\text { HRF, } n \text { (\% } \% \text { SE } \\
\text { Eyes) }\end{array}$} & \multirow{2}{*}{$\begin{array}{l}\text { Adjuvant IVC, } \\
n(\%) \text { Es Eyes } \\
\text { with HRF) }\end{array}$} & \multirow{2}{*}{$\begin{array}{c}\text { Mean/Median } \\
\text { Follow-Up, } \\
\text { Months (Range) }\end{array}$} & \multirow{2}{*}{$\begin{array}{l}\text { Orbit Invasion, } \\
n \text { (\% Patients) }\end{array}$} & \multirow{2}{*}{$\begin{array}{l}\text { Metastases, } n \\
(\% \text { Patients) }\end{array}$} & \multirow{2}{*}{$\begin{array}{c}\text { Deaths, } n \\
\text { (\% Patients) }\end{array}$} \\
\hline & & All & SE Eyes & All & SE Eyes & All & SE Eyes $^{++}$ & & & & & & & & \\
\hline $\begin{array}{l}\text { Alkatan et al. } \\
(2020)[277\end{array}$ & 2013-2017 & - & $28 / 26$ & - & $\begin{array}{c}\text { ICRB: } \\
D+E=28\end{array}$ & - & $\begin{array}{l}\text { IVC }=27 \\
\text { EBRT }=14\end{array}$ & Active disease $=28(100)^{*}$ & $8(0-38)$ & $6(21)$ & $6(21)^{y}$ & na & $1(4)^{\mathrm{z}}$ & $3(12)^{\mathrm{z}}$ & $3(12)^{\mathrm{z}}$ \\
\hline
\end{tabular}

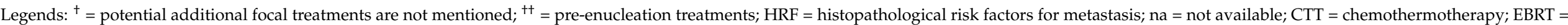

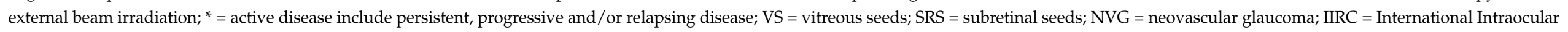

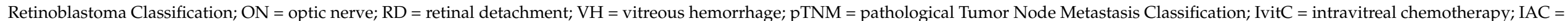

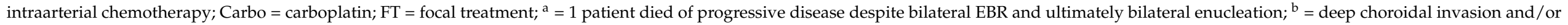

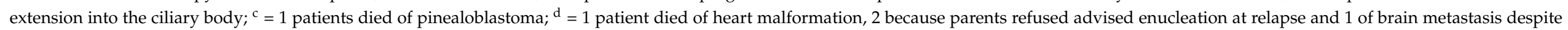

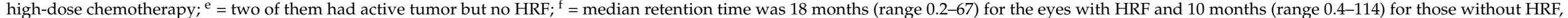

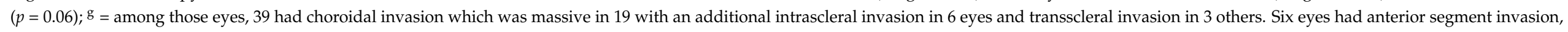

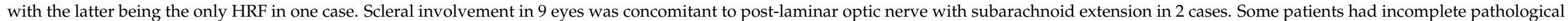

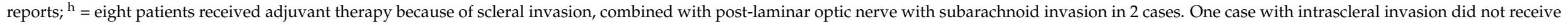

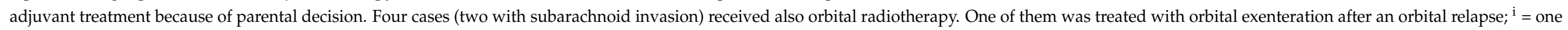

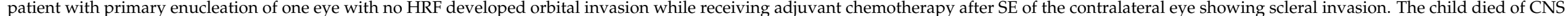

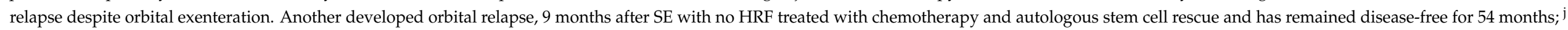

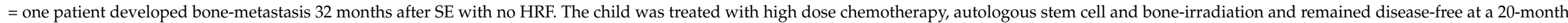

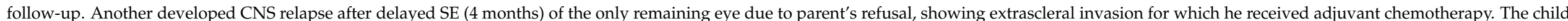

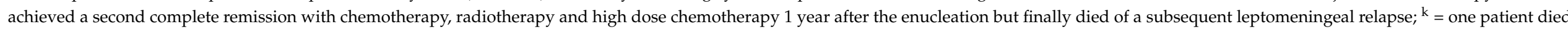

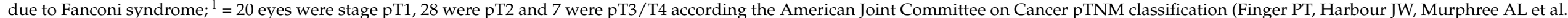

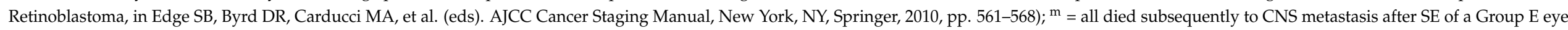

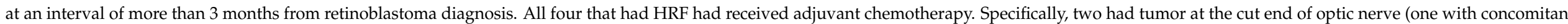

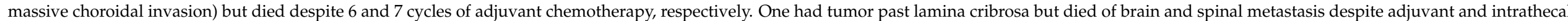

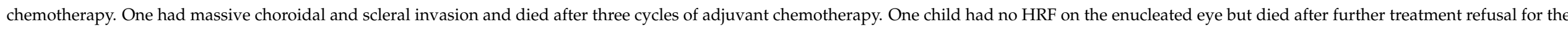

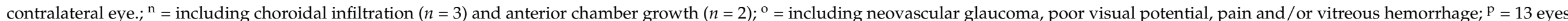

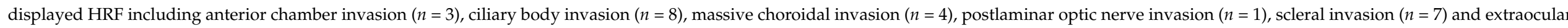

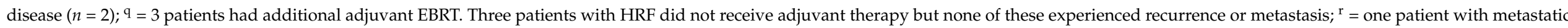

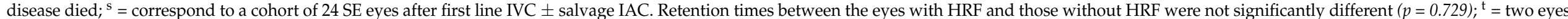

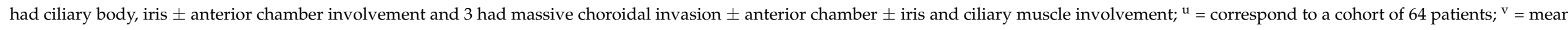

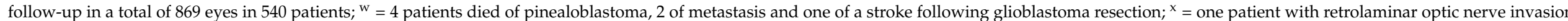

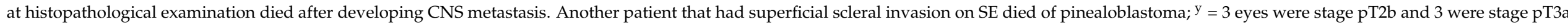

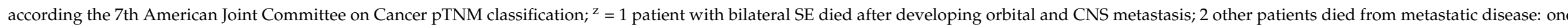

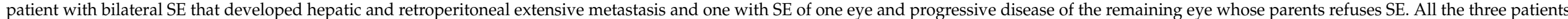
who died had at least one secondary enucleated eye with HRF. 
Table 3. Secondary enucleations (SE), orbital invasion, metastases and mortality rates after first line $\left(1^{\circ}\right)$ or salvage intra-arterial $\left(2^{\circ}\right)$ chemotherapy (IAC): literature review.

\begin{tabular}{|c|c|c|c|c|c|c|c|c|c|c|c|c|c|c|c|}
\hline \multirow{2}{*}{$\begin{array}{c}\text { Author } \\
\text { (Year) }\end{array}$} & \multirow{2}{*}{ Study Years } & \multicolumn{2}{|c|}{ Eyes/Patients } & \multicolumn{2}{|c|}{ Classification Group } & \multicolumn{2}{|c|}{ Treatments +} & \multirow{2}{*}{$\begin{array}{c}\text { Indication for SE, } \\
n(\% \text { SE Eyes) }\end{array}$} & \multirow{2}{*}{$\begin{array}{c}\text { Mean Reten- } \\
\text { tion Time, } \\
\text { Months (Range) } \\
\end{array}$} & \multirow{2}{*}{$\begin{array}{c}\text { HRF, } \\
n \text { (\% SE Eyes) }\end{array}$} & \multirow{2}{*}{$\begin{array}{l}\text { Adjuvant IVC, } \\
n \text { (\% SE Eyes } \\
\text { with HRF) }\end{array}$} & \multirow{2}{*}{$\begin{array}{c}\text { Mean/Median } \\
\text { Follow-Up, } \\
\text { Months (Range) }\end{array}$} & \multirow{2}{*}{$\begin{array}{c}\text { Orbit } \\
\text { Invasion, } \\
n(\% \text { Patients) } \\
\end{array}$} & \multirow{2}{*}{$\begin{array}{l}\text { Metastases, } n \\
\text { (\% Patients) }\end{array}$} & \multirow{2}{*}{$\begin{array}{r}\text { Deaths, } n \\
\text { (\% Patients }\end{array}$} \\
\hline & & All & SE Eyes & All & SE Eyes & All & SE Eyes * & & & & & & & & \\
\hline $\begin{array}{l}\text { Abramson et al. } \\
\text { (2012) [28] }\end{array}$ & 2006-2011 & 30/na & $1 / 1$ & $\begin{array}{l}\text { COG: } \\
\text { B }=19 \\
\text { C }=11\end{array}$ & $\begin{array}{l}\text { COG: } \\
\mathrm{C}=1\end{array}$ & $\begin{array}{l}\mathrm{na}=29 \\
2^{\circ}=1\end{array}$ & IAC: $2^{\circ}=1$ & $\begin{array}{l}\text { 2nd IAC not feasible for } \\
\text { technical reason }=1(100)\end{array}$ & na & na & na & $16(3-33)$ & $0(0)$ & $0(0)$ & $0(0)$ \\
\hline $\begin{array}{l}\text { Palioura et al. } \\
\text { (2012) [29] }\end{array}$ & 2006-2010 & $37 / 34$ & $5 / 5$ & $\begin{array}{l}\text { COG: } \\
D=31 \\
E=6\end{array}$ & na & $\begin{array}{c}\text { IAC: } 1^{\circ}=27 \\
2^{\circ}=10 \\
\text { IV }=9 \\
\text { EBRT }=3\end{array}$ & $\begin{array}{c}\text { IAC: } 1^{\circ}=3 \\
2^{0}=2 \\
I V C=2 \\
\text { EBRT }=1\end{array}$ & $\begin{array}{l}\text { Active tumor }=4(80) \\
\text { Tractional } \mathrm{RD}=1(20)\end{array}$ & $\mathrm{na}^{\mathrm{a}}$ & na & na & $21(1-42)$ & $0(0)$ & $1(3)^{\mathrm{b}}$ & $0(0)$ \\
\hline $\begin{array}{l}\text { Thampi et al. } \\
\text { (2013) [30] }\end{array}$ & 2010-2012 & $20 / 16$ & $6 / 6$ & $\begin{array}{l}\text { ICRB: } \\
\mathrm{A}=1 \\
\mathrm{~B}=4 \\
\mathrm{C}=2 \\
\mathrm{D}=11 \\
\mathrm{E}=2\end{array}$ & $\begin{array}{l}\text { ICRB: } \\
D=5 \\
E=1\end{array}$ & $\begin{aligned} \operatorname{IAC}: 1^{1} & =12 \\
2^{0} & =8 \\
\mathrm{IVC} & =8\end{aligned}$ & $\begin{array}{l}\mathrm{IAC} 1^{\circ}=5 \\
\mathrm{IAC} 2^{\circ}=1 \\
\mathrm{EBRT}=1 \\
\text { IVC }=2\end{array}$ & $\begin{array}{c}\text { Active tumor }=6(100) \\
-\mathrm{VH} \text { obscuring fundus } \\
\text { view }=2\end{array}$ & na & $1(17)^{\mathrm{c}}$ & $2(100)^{d}$ & $\begin{array}{l}\text { All: } \\
\text { 15(1-29) } \\
\text { SE group: } \\
15(6-29)\end{array}$ & $0(0)$ & $0(0)$ & $0(0)$ \\
\hline $\begin{array}{l}\text { Shields et al. } \\
(2013)[11]\end{array}$ & 2008-2011 & $14 / 14$ & $6 / 6$ & $\begin{array}{l}\text { ICRB: } \\
D=6 \\
\mathrm{E}=8\end{array}$ & $\begin{array}{l}\text { ICRB: } \\
\mathrm{D}=2 \\
\mathrm{E}=4\end{array}$ & $\begin{array}{c}\text { IAC: } \\
2^{\circ}=14 \\
\mathrm{IVC}=14\end{array}$ & $\begin{array}{l}\text { IAC: } \\
2^{\circ}=6\end{array}$ & $\begin{array}{l}\text { Active tumor }=6(100) \\
-\mathrm{RT}=2 \\
-\mathrm{SRS}+/-\mathrm{VS}=3 \\
- \text { suspicion } \mathrm{ON} \text { invasion on } \\
\text { MRI } 1\end{array}$ & na & $0(0)$ & $0(0)$ & $\begin{array}{l}\text { All: } \\
\text { 24(17,5-48) } \\
\text { SE group: } \\
19(9-36)\end{array}$ & $0(0)$ & $0(0)$ & $0(0)$ \\
\hline $\begin{array}{l}\text { Venturi et al. } \\
\text { (2013) [32] }\end{array}$ & 2008-2010 & $39 / 36$ & $8 / 8$ & $\begin{array}{l}\text { TNM: } \\
1 \mathrm{a}=1 \\
1 \mathrm{~b}=12 \\
2 \mathrm{a}=8 \\
2 \mathrm{~b}=14 \\
3 \mathrm{a}=4\end{array}$ & $\begin{array}{c}\text { RE: } \\
\mathrm{Vb}=8\end{array}$ & $\begin{array}{c}\text { IAC: } \\
1^{\circ}=17 \\
2^{\circ}=22 \\
\text { IVC }=21 \\
\text { EBRT }=1 \\
\text { Plaque }=1 \\
\text { IvitC }=1\end{array}$ & $\begin{array}{l}\text { IAC: } \\
1^{\circ}=7 \\
2^{\circ}=1\end{array}$ & Active tumor $=8(100)$ & na & $\begin{array}{l}\mathrm{pT1}=6(75) \\
\mathrm{pT3a}=1(12) \\
\mathrm{na}=1(12)^{\mathrm{e}}\end{array}$ & na & $13(1-27)^{\mathrm{f}}$ & $0(0)^{\mathrm{f}}$ & $0(0){ }^{\mathrm{f}}$ & $0(0)^{\mathrm{f}}$ \\
\hline $\begin{array}{l}\text { Bracco et al. } \\
\text { (2013) [33] }\end{array}$ & 2008-na & $52 / 47$ & $18 / \mathrm{na}$ & $\begin{array}{l}\text { IIIRC: } \\
A=5 \\
B=18 \\
C=4 \\
D=25\end{array}$ & $\begin{array}{c}\text { IIRC: } D=12 \\
\text { na }=6\end{array}$ & $\begin{array}{c}\text { IAC: } \\
1^{\circ}=22 \\
2^{\circ}=30 \\
\text { IVC }=30 \\
\text { EBRT }=1 \\
\text { Plaque }=1 \\
\text { IvitC }=1\end{array}$ & $\begin{array}{l}\text { IAC: } \\
1^{\circ}=12 \\
2^{\circ}=6\end{array}$ & na & па & na & na & $40(19-61)^{\mathrm{f}}$ & $0(0)^{\mathrm{f}}$ & $0(0)^{\mathrm{f}}$ & $O(0)^{\mathrm{f}}$ \\
\hline $\begin{array}{l}\text { Shields et al. } \\
(2014)[34]\end{array}$ & 2009-2013 & $70 / 70$ & $23 / 23$ & $\begin{array}{l}\text { ICRB: } \\
B=1 \\
\text { C }=4 \\
D=17 \\
\text { E }=14 \\
\text { na }=34\end{array}$ & $\begin{array}{c}\text { ICRB: } \\
D=1 \\
\mathrm{E}=9 \\
\text { na }=13\end{array}$ & $\begin{array}{c}\text { IAC: } \\
1^{\circ}=36 \\
2^{\circ}=34 \\
\text { IVC }=34\end{array}$ & $\begin{array}{c}\text { IAC: } \\
1^{\circ}=10 \\
2^{\circ}=13\end{array}$ & $\begin{array}{c}\text { After IAC } 1^{\circ}: \text { na }=10 \\
\text { After IAC } 2^{\circ}: \\
\text { Active tumor }=10(77) \\
\text { VH }=2(15) \\
\text { NVG }=1(8)\end{array}$ & nа & na & na & 19 (na) & $0(0)$ & $0(0)$ & $0(0)$ \\
\hline $\begin{array}{l}\text { Ong et al. (2015) } \\
\text { [35] }\end{array}$ & 2010-2013 & $17 / 12$ & $7 / 6$ & $\begin{array}{l}\text { ICRB: } \\
B=3 \\
C=1 \\
D=1 \\
\mathrm{E}=12\end{array}$ & $\begin{array}{l}\text { ICRB: } \\
B=1 \\
E=6\end{array}$ & $\begin{array}{c}\text { IAC: } \\
1^{\circ}=6 \\
2^{\circ}=11 \\
\text { IVC }=11 \\
\text { EBRT }=4 \\
\text { IvitC }=3\end{array}$ & $\begin{array}{c}\text { IAC: } \\
1^{\circ}=2 \\
2^{\circ}=5 \\
\text { EBRT }=1\end{array}$ & $\begin{array}{c}\text { Active tumor }=5(71) \\
-\mathrm{RT}+\mathrm{VS} \pm \mathrm{VH} \pm \mathrm{RD}=3 \\
-\mathrm{VS}+\mathrm{VH}=1 \\
-\mathrm{NVG}+\text { corneal haze }=1 \\
\mathrm{VH}=2(29)\end{array}$ & nа & $3(43) \mathrm{g}$ & $3(100)^{g}$ & $\begin{array}{l}\text { All: } \\
\text { 21 (5-43) } \\
\text { SE group: } \\
\text { 22 (12-35) }\end{array}$ & $0(0) \mathrm{g}$ & $3(25) \mathrm{g}$ & $2(17)^{g}$ \\
\hline $\begin{array}{c}\text { Yannuzzi et al. } \\
\text { (2015) [36] }\end{array}$ & 2006-2014 & $77 / 72$ & $10 / \mathrm{na}$ & $\begin{array}{l}\text { COG: } \\
C=2 \\
D=52 \\
E=23\end{array}$ & na & $\begin{array}{l}\text { IAC: } \\
1^{\circ}=77\end{array}$ & $\begin{array}{l}\text { IAC: } \\
1^{\circ}=10\end{array}$ & na & na & $1(10)^{\mathrm{h}}$ & na & $39(9-104)$ & $1(1)^{\mathrm{i}}$ & $3(4)^{\mathrm{i}}$ & $0(0)^{\mathrm{i}}$ \\
\hline $\begin{array}{l}\text { Akyuz et al. } \\
\text { (2015) [37] }\end{array}$ & 2011-2014 & $56 / 46$ & $19 /$ na & $\begin{array}{l}\text { ICRB: } \\
\mathrm{A}=7 \\
\mathrm{~B}=6 \\
\mathrm{C}=16 \\
\mathrm{D}=19 \\
\mathrm{E}=8\end{array}$ & na & $\begin{array}{c}\text { IAC: } \\
1^{\circ}=12 \\
2^{\circ}=44 \\
\text { IVC }=44\end{array}$ & $\begin{array}{l}\text { IAC: } \\
1^{\circ}=3 \\
2^{\circ}=16\end{array}$ & $\begin{aligned} & \text { Active tumor }=19(100) \\
-V S & =10 \\
- & n a=9\end{aligned}$ & na & $6(32)^{\mathrm{j}}$ & $6(100)^{j}$ & $12(1-28)$ & $1(2)^{\mathrm{j}}$ & $1(2)^{\mathrm{j}}$ & $2(4)^{\mathrm{j}}$ \\
\hline
\end{tabular}


Table 3. Cont.

\begin{tabular}{|c|c|c|c|c|c|c|c|c|c|c|c|c|c|c|c|}
\hline \multirow{2}{*}{$\begin{array}{c}\text { Author } \\
\text { (Year) }\end{array}$} & \multirow{2}{*}{ Study Years } & \multicolumn{2}{|c|}{ Eyes/Patients } & \multicolumn{2}{|c|}{ Classification Group } & \multicolumn{2}{|c|}{ Treatments ${ }^{\prime}$} & \multirow{2}{*}{$\begin{array}{c}\text { Indication for SE, } \\
n(\% \text { SE Eyes })\end{array}$} & \multirow{2}{*}{$\begin{array}{c}\text { Mean Reten- } \\
\text { tion Time, } \\
\text { Months (Range) }\end{array}$} & \multirow{2}{*}{$\begin{array}{c}\text { HRE, } \\
n \text { (\% SE Eyes) }\end{array}$} & \multirow{2}{*}{$\begin{array}{c}\text { Adjuvant IVC, } \\
n(\% \text { SE Eyes } \\
\text { with HRF) }\end{array}$} & \multirow{2}{*}{$\begin{array}{c}\text { Mean/Median } \\
\text { Follow-Up, } \\
\text { Months (Range) }\end{array}$} & \multirow{2}{*}{$\begin{array}{c}\text { Orbit } \\
\text { Invasion, } \\
n \text { (\% Patients) }\end{array}$} & \multirow{2}{*}{$\begin{array}{l}\text { Metastases, } n \\
\text { (\% Patients) }\end{array}$} & \multirow{2}{*}{$\begin{array}{r}\text { Deaths, } n \\
\text { \% Patients }\end{array}$} \\
\hline & & All & SE Eyes & All & SE Eyes & All & SE Eyes * & & & & & & & & \\
\hline $\begin{array}{l}\text { Abramson et al. } \\
(2016)[38]\end{array}$ & 2006-2012 & $112 / 103$ & $24 / \mathrm{na}$ & $\begin{array}{l}\text { COG: } \\
\mathrm{D}=112\end{array}$ & $\begin{array}{l}\text { COG: } \\
\mathrm{D}=24\end{array}$ & $\begin{array}{c}\text { IAC: } \\
1^{\circ}=54 \\
\text { IVC }=7 \\
2^{\circ}=58 \\
\text { IVC }=51 \\
\text { EBRT }=15 \\
\text { Plaque }=4\end{array}$ & $\begin{array}{c}\text { IAC: } \\
1^{\circ}=8 \\
2^{\circ}=16 \\
\text { EBRT }+16=5 \\
\text { EBRT }=1 \\
\text { IVC }=9 \\
\text { Plaque }=1\end{array}$ & na & па & na & na & $34(2-110)$ & $0(0)$ & $3(6)^{\mathrm{k}}$ & $1(2)^{1}$ \\
\hline $\begin{array}{l}\text { Abramson } \\
\text { et al. (2016) [39] }\end{array}$ & 2008-2015 & 120/60 & $4 / 4$ & $\begin{array}{l}\text { COG: } \\
\mathrm{A}=2 \\
\mathrm{~B}=18 \\
\mathrm{C}=24 \\
\mathrm{D}=56 \\
\mathrm{E}=20\end{array}$ & $\begin{array}{l}\text { COG } \\
D=2 \\
E=2\end{array}$ & $\begin{array}{l}\text { IAC: } \\
1^{\circ}=30 \\
2^{\circ}=30\end{array}$ & $\begin{array}{l}\text { IAC: } \\
1^{\circ}=2 \\
2^{\circ}=2\end{array}$ & $\begin{aligned} \text { Active tumor } & =3(75) \\
\text { Parental choice } & =1(25)\end{aligned}$ & na & na & na & na & $0(0)$ & $0(0)$ & $1(2) \mathrm{m}$ \\
\hline $\begin{array}{l}\text { Chen et al. } \\
\text { (2017) [41] }\end{array}$ & 2011-2013 & $107 / 73$ & $23 / \mathrm{na}$ & $\begin{array}{l}\text { IIRC: } \\
\text { B }=11 \\
\mathrm{C}=11 \\
\mathrm{D}=56 \\
\mathrm{E}=29\end{array}$ & $\begin{array}{l}\text { IIRC: } \\
\mathrm{D}=12 \\
\mathrm{E}=11\end{array}$ & $\begin{array}{c}\text { IAC: } \\
1^{\circ}=30 \\
2^{\circ}=77 \\
I V C=64\end{array}$ & $\begin{array}{c}\text { IAC: } \\
1^{\circ}=9 \\
2^{\circ}=13\end{array}$ & $\begin{array}{c}\text { Active tumor }=19(83) \\
-R T=12 \\
-V S=4 \\
-S R S=3 \\
\quad V H=4(17)\end{array}$ & na & na & na & $14(3-28)$ & па & na & na \\
\hline $\begin{array}{l}\text { Funes et al. } \\
\text { (2018) [42] }\end{array}$ & 2010-2015 & $97 / 81$ & $31 / \mathrm{na}$ & $\begin{array}{l}\text { IIRC: } \\
\text { B }=5 \\
C=8 \\
D=22 \\
\text { na }=62\end{array}$ & na & $\begin{array}{c}\text { IAC: } \\
1^{1}=35 \\
I V C=12 \\
2^{\circ}=62 \\
I V C=62 \\
E B R T=12\end{array}$ & $\begin{array}{c}\text { IAC: } \\
1^{\circ}=11 \mathrm{~m} \\
2^{\circ}=20\end{array}$ & na & na & $3(10)^{\mathrm{n}}$ & $3(100)^{n}$ & $49(12-72)$ & $2(2)$ & $0(0)$ & $2(2)^{\circ}$ \\
\hline
\end{tabular}

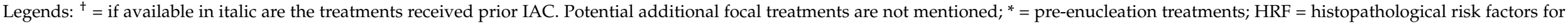
metastasis; na = not available; COG = Children's Oncology Group; EBRT = external beam radiotherapy; IVC = intravenous chemotherapy; ICRB = International Classification for Retinoblastoma; RD = retinal detachment; RT = retinal tumor; SRS = subretinal seeds; VS = vitreous seeds; ON = optic nerve; MRI = magnetic resonance imagery; RE = Reese-Ellsworth Classification; TNM = Tumor Node Metastasis Staging; pTNM; pathological TNM; VH = vitreous hemorrhage; NVG = neovascular glaucoma; IIRC = International Intraocular Retinoblastoma Classification; ${ }^{\mathrm{a}}=$ estimated 0-6 month-interval

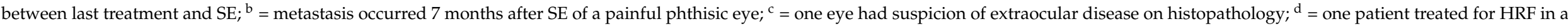
non-IAC treated eye; ${ }^{\mathrm{e}}=$ one patient had SE elsewhere; ${ }^{\mathrm{f}}=$ two patients were lost to follow-up; ${ }^{\mathrm{g}}=$ three SE eyes after IAC $2^{\circ}$ had high-risk features (choroid and optic nerve invasion) and developed systemic metastasis despite receiving adjuvant chemotherapy. Two of them died; ${ }^{\mathrm{h}}=$ one SE eye had histopathological features defined as optic nerve invasion post lamina cribrosa, massive choroidal invasion

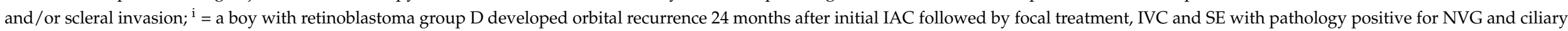
body invasion. Subsequent work-up revealed metastatic disease. The patient is still alive without signs of metastases, 84 months after initial presentation. Mean time to metastatic disease from initial treatment was 26 months; $^{j}=$ pathological risk factors are not detailed in that study. All SE patients with HRF had undergone previous second line IAC. Two of them (one with histopathological anterior chamber and choroidal invasion and one with optic nerve invasion) died due to progressive disease despite multimodal treatment after enucleation; ${ }^{k}=$ three patients treated with first line IAC developed metastases. All were successfully treated; ${ }^{1}=$ one patient died of pinealoblastoma; ${ }^{\mathrm{m}}=2$ patients were treated with IAC until parents agreed to enucleate. One eye showed 1 massive choroid, the other had no

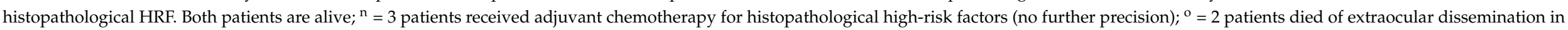
the context of refusal of timely enucleation. 


\section{Results}

\subsection{Shift of SE Indications over the Years}

Since the beginning of conservative retinoblastoma management, the main reason to stop eye-preserving therapies has always been, and still is related, independently of the treatment modalities used, to uncontrolled tumor activity (80-90\% of SE) $[2,4-7,10,11,13-24,26,27,29-32,34,35,37,38,40,41,53-56]$, while the occurrence of intraocular complications, especially those obscuring the fundus view, explains the remaining cases (10-20\% of SE) $[2,5,10,11,13,16-18,21,23,28,29,34,39-41]$ (see Tables 1-3). Constant innovations in diagnostic and therapeutic retinoblastoma care have, however, allowed progressive improvement of tumor control and successful treatment of various complications that would otherwise compromise globe-salvage, leading, over the years, to a continuous shift in SE indications and a significant decrease of the overall SE rate [57].

\subsection{The Role of Intra-Arterial, Intravitreal and Intracameral Chemotherapy and Management of Treatment-or Tumor-Related Complications}

The greatest advance in the eye-preserving treatment of retinoblastoma has undeniably been brought about by the development of various techniques to safely deliver high chemotherapy drug concentrations into the different eye compartments, allowing an unprecedented control of both solid tumors and seeding [57]. First of all, the modern approach of super-selective intra-arterial chemotherapy introduced in 2008 [58] enabled not only to salvage heavily pre-treated eyes that would otherwise have faced SE, but also to achieve a higher control of retinal tumor $(92 \%$ versus $62 \%, p=0.002)$ and subretinal seeding ( $86 \%$ versus $31 \%, p=0.006$ ), compared to intravenous chemotherapy for advanced treatment-naïve retinoblastoma groups D and E [59]. Furthermore, intra-arterial chemotherapy was also shown to be effective in isolated cases of massive choroidal $[60,61]$ and iris invasion [62], but not in ciliary body invasion requiring brachytherapy for tumor control $[57,63]$, with no reported metastasis nor deaths, indicating that posterior and anterior uveal involvement should not necessarily be considered as an absolute criterion for enucleation, nor a definite indication for adjuvant chemotherapy [64-66]. In 2012, the introduction of a safety-enhanced technique to perform intravitreal injection in an eye with active tumor [67] permitted almost absolute control (close to $100 \%$ of the cases) of the vitreous disease [43,57,68-72], previously leading to SE in about $50 \%$ of the eyes managed with first line external beam irradiation or chemoreduction $[9,46]$ and $36 \%$ of those managed with first line intra-arterial chemotherapy [73]. Noteworthy, both intravitreal [74] and/or intraarterial injections $[75,76]$ can be successfully repeated in relapsing cases. In addition to its use for active tumor treatment, intravitreal melphalan has also enabled a more secure management of various complications, necessitating intraocular surgery such as cataract [57] or tractional retinal detachment [77-79]. Similarly, the use of intravitreal anti-VEGF injections performed according to the same technique as intravitreal chemotherapy has permitted the eye-preserving management of treatment- or tumor-related neovascular complications, earlier commonly treated with immediate enucleation [80]. Finally, the more recent inception in 2015 of a technique adapted to inject safely into the anterior and posterior chambers, namely intracameral chemotherapy $[81,82]$, has shown promising results for the control of aqueous seeding $[57,83,84]$, previously also treated with immediate enucleation.

While the use of intra-arterial, intravitreal and intracameral chemotherapies is considered safe, with no reported life-threatening related adverse effects $[62,85,86]$, their wide implementation in retinoblastoma management has raised concerns on the possible negative consequences of a lower systemic chemotherapy exposure and its potential benefit in preventing systemic metastasis in children with microscopically-undetected disseminated disease who relapse with metastatic disease following completion of treatment $[87,88]$. Although more studies with longer follow-ups are needed to be able to reach a conclusion, such fears have not, however, been confirmed to date. Indeed, when considering the studies reporting on metastatic disease, a similar metastasis rate of about $2 \%$ is found in children treated with systemic chemotherapy $[15,16,19,22,25,26]$ or intra-arterial 
chemotherapy $[23,29,33-35,37,38,42]$, whereas, according to a retrospective multicentric survey including more than 1100 patients managed with primary $(n=464)$ or salvage intra-arterial chemotherapy $(n=713)$ over a 10-year period, the risk of metastatic death from retinoblastoma has been estimated to be less than $1 \%$ [89].

\subsection{The Role of Ancillary Testing}

Along with the advances brought about by the emergence of new treatment modalities, the development of various imaging techniques aiming at evaluating the disease extent (especially if there is fear of exteriorization or in case of fundus view loss) as well as the presence or not of tumor activity has been crucial to set up the limits of eye-preserving treatment. Thus, spectral domain optical coherence tomography (OCT) has been instrumental in the eye-preserving management of cases with choroidal [60] or epipapillary relapse [90] by allowing early tumor detection and close monitoring of the treatment response. Recently, anterior segment OCT has also been reported to accurately detect in vivo tumor progression into the Schlemm's canal [91]. Fluorescein angiography allows to assess the tumoral and retinal vascularization status [92] and to monitor the treatment response to intraocular vascular complications [80]. In the case of opaque media, ultrasonography (B-scan) provides useful information to assess any tumor growth or optic nerve threat, while high-resolution contrast-enhanced magnetic resonance imaging (MRI) enables the evaluation of tumor activity and potential exteriorization. While its sensitivity and specificity in detecting scleral and peribulbar invasion is considered to be near $100 \%$, MRI is, however, less sensitive to detecting early choroidal [93] or postlaminar optic nerve invasion $[93,94]$. Finally ultrasonic biomicroscopy proved to be instrumental, not only for the determination of a tumor-free entry meridian prior to intravitreal injection for vitreous disease in case of compromised pupil dilation or presence of opaque media [57,71], but also for the assessment and monitoring of tumor invasion of the ciliary body and/or posterior chamber $[57,81]$.

\subsection{Influence of Delayed Enucleation on Metastasis Rate and Survival}

The advent of the afromentioned diagnostic and therapeutic modalities resulted in more advanced diseased eyes and more heavily pretreated ones escaping primary and SE respectively, raising concerns of a potential negative impact on metastasis rate and overall survival in case of delayed enucleation [87]. In a series of 45 group E eyes enucleated for persistent disease after first line systemic chemotherapy, the authors reported that $\mathrm{SE}$ delayed for more than three months after diagnosis was associated with mortality in four patients as a result of pathologic downstaging of the disease and reduced surveillance leading to inappropriate management of unrecognized high-risk factor for metastasis [19]. In two studies comparing histopathology in eyes treated with first line enucleation versus SE, others demonstrated, however, that prolonged times to enucleation were associated with the presence of high-risk features but not to the development of metastasis nor mortality $[21,24]$, suggesting that prompt recognition of refractory disease followed by timely enucleation and adjuvant therapy for high risk factors can efficiently prevent metastatic dissemination [21]. Interestingly, in a study including 24 eyes enucleated after chemoreduction at an average time of five months after loss of fundus view, $22(92 \%)$ had viable tumor cells on histopathology, but none of them showed high-risk features [55]. Finally, in two other studies comparing advanced retinoblastoma group D/E treated with either first line systemic chemotherapy or first line intra-arterial chemotherapy versus primary enucleation, conservative treatment was not found to increase the risk of orbital recurrences, metastatic disease or death [22,36].

\subsection{Indication for SE and Management of High-Risk Pathologic Factors}

The establishment of clear guidelines regarding the optimal timing of SE and the need for post-enucleation adjuvant chemotherapy is hindered by the present lack of studies having focused on that subject with only little information available from the studies 
reporting their treatment outcomes (especially regarding retention times or management of cases with loss of fundus view, and clinicopathologic correlations) and the overall low rates of metastatic disease. On the other hand, the absence of a consensus for the definition of high-risk pathologic factors, with some considering anterior chamber invasion or isolated massive choroidal invasion as a high-risk features for metastasis [95], while others not $[64,96,97]$, as well as considerable variations in the use and type of post-enucleation adjuvant therapies precludes any conclusions regarding the metastasis risk and comparison of survival rates [95]. The use of the recently-proposed classification of retinoblastoma at relapse (RSU classification), which aims to standardize the treatment for relapse based on the recurrence localization [57], and the classification of regressed retinoblastoma (RBRecist) [98] should allow a better comparison of treatment outcomes and help define SE criteria in the coming years. Last but not least, the future validation of tumor-specific biomarkers in liquid biopsies may revolutionize the conservative retinoblastoma management by stratifying the risk of metastasis in a histopathologic-independent manner and/or diagnosing minimally disseminated disease in blood, cerebrospinal fluid or bone marrow [99].

In the expectation of prospective studies that could bring evidence-based answers to the above concerns, on behalf of the European Retinoblastoma Group (EURbG), we propose guidelines to assist decision making with respect to when SE becomes imperative (absolute indication) or recommended (relative indication for SE) as a result of a consensus based on the clinical experience of each co-author active in European referral center and the above discussed review of the literature (see Table 4). 
Table 4. Guidelines for secondary enucleation (SE) in the retinoblastoma treatment *.

Conditions where maintaining an eye is considered to be life-threatening or associated with untreatable to be life-threatening or as

Absolute indications are independent of the disease laterality
(1) Refractory disease despite salvage treatment Direct or indirect sign(s) of disease exteriorization

Absolute indications for SE
(3) Neovascular complications associated with untreatable ischemic retinopathy

(4) Phthisis bulbi
Suspicion of loss of tumor control should motivate enucleation within 7-14 days

Pre-enucleation MRI in case of loss of fundus view or suspicion of disease exteriorization

Histopathological analysis for evaluation of high-risk factors for metastasis should be performed in all SE cases
Conditions where SE are may be indicated but where eye-preserving management could be considered, especially if it is the only seeing eye provided that certain criteria are met including:

parental full awareness of the residual risk of metastasis and death

guarantee that appropriate follow-up can be undertaken

availability of advanced diagnostic and therapeutic modalities in the treating centre
(1) Regressed tumor covering the optic nerve

(2) Obscured tumor view secondary to poo pupillary dilatation, cataract, loss of corneal transparency and/o

Relative indications for SE

intravitreal haemorrhage
(3) Tractional or rhegmatogenous

retinal detachment

(4) Neovascular complications (neovascular

glaucoma, retinal and/or

papillary neovascularization)
In the case of a regressed tumor covering the optic nerve, patients should be followed with a 1.5 or 3 Tesla MRI, every 3 months for one year after treatment completion, then every 4 months for one year then every 6 months for at least one year Eyes with a regressed tumor covering the optic nerve after systemic chemoreduction may be consolidated with 2 courses of intra-arterial chemotherapy.

- $\quad$ Eyes with ocular complications such as poor pupillary dilatation, cataract, tractional or rhegmatogenous retinal detachment, loss of corneal transparency, and/or intravitreal detachment, loss of corneal transparency, and/or intravitreal
hemorrhage should also be followed with regular appropriate imaging until the fundus view is spontaneously or surgically restored. (NB: Medical and surgical recommendations for the management of intraocular complications are beyond the scope of this article but should be given based on the available literature). Histopathological analysis for evaluation of high-risk factors for metastasis should be performed in all SE cases.

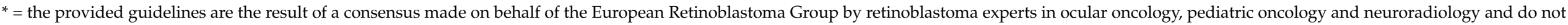
reflect any evidence-based recommendations. 


\section{Conclusions}

Despite a growing use of conservative treatments, SE still has a central role to play in the management of retinoblastoma to preserve the patient from metastasis and death. Although the need for SE cannot, to date, be unequivocally delineated, it is however possible to draw distinction borders between absolute and relative indications for SE depending on the available diagnostic and therapeutic modalities and on consensus among the local multidisciplinary retinoblastoma team (Table 4). Thus, absolute indications may be restricted to eyes with refractory tumor activity resisting all therapeutic modalities or eyes under apparent tumor control but no visual potential and untreatable intra-ocular complications. In contrast, eyes with an obscured optic nerve head and/or ocular complications amenable to specific surgical or medical management can be considered relative indications to SE or may be conditionally maintained, provided that appropriate follow-up can be implemented and that parents are fully aware of a residual risk.

Author Contributions: The authors contributed as follows: conceptualization: C.S., L.L.-L.R., A.C.M., F.D., G.C., M.P., H.J., M.B.P., F.L.M.; writing—original draft preparation: C.S. review: L.L.-L.R., A.C.M., F.D., G.C., M.B.P., M.P., H.J., P.M., F.L.M.; editing: C.S., F.L.M.; supervision: C.S., F.L.M. All authors have read and agreed to the published version of the manuscript.

Funding: This research received no external funding.

Institutional Review Board Statement: The study did not require ethical approval.

Informed Consent Statement: No applicable.

Data Availability Statement: Data sharing not applicable. No new data were created or analyzed in this study.

Conflicts of Interest: The authors declare no conflict of interest.

\begin{tabular}{ll}
\multicolumn{2}{l}{ Abbreviations } \\
SE & secondary enucleation \\
MRI & $\begin{array}{l}\text { Magnetic resonance imaging } \\
\text { optical coherence tomography }\end{array}$ \\
OCT & European Retinoblastoma Group \\
EURbG & optical coherence tomography \\
OCT & anti-vascular endothelial growth factor \\
anti-VEGF
\end{tabular}

\section{References}

1. Thompson, R.W.; Small, R.C.; Stein, J.J. Treatment of retinoblastoma. Am. J. Roentgenol. Radium Ther. Nucl. Med. 1972, 114, 16-23.

2. Egbert, P.R.; Donaldson, S.S.; Moazed, K.; Rosenthal, A.R. Visual results and ocular complications following radiotherapy for retinoblastoma. Arch. Ophthalmol. 1978, 96, 1826-1830. [CrossRef]

3. Foote, R.L.; Garretson, B.R.; Schomberg, P.J.; Buskirk, S.J.; Robertson, D.M.; Earle, J.D. External beam irradiation for retinoblastoma: Patterns of failure and dose-response analysis. Int. J. Radiat. Oncol. Biol. Phys. 1989, 16, 823-830. [CrossRef]

4. Toma, N.M.; Hungerford, J.L.; Plowman, P.N.; Kingston, J.E.; Doughty, D. External beam radiotherapy for retinoblastoma: II. Lens sparing technique. Br. J. Ophthalmol. 1995, 79, 112-117. [CrossRef]

5. Hernandez, J.C.; Brady, L.W.; Shields, J.A.; Shields, C.L.; De Potter, P.; Karlsson, U.L.; Markoe, A.M.; Amendola, B.E.; Singh, A. External beam radiation for retinoblastoma: Results, patterns of failure, and a proposal for treatment guidelines. Int. J. Radiat. Oncol. Biol. Phys. 1996, 35, 125-132. [CrossRef]

6. Blach, L.E.; McCormick, B.; Abramson, D.H. External beam radiation therapy and retinoblastoma: Long-term results in the comparison of two techniques. Int. J. Radiat. Oncol. Biol. Phys. 1996, 35, 45-51. [CrossRef]

7. Merchant, T.E.; Gould, C.J.; Hilton, N.E.; Kun, L.E.; Rodriguez-Galindo, C.; Pratt, C.B.; Wilson, M.W.; Haik, B. Ocular preservation after 36 Gy external beam radiation therapy for retinoblastoma. J. Pediatr. Hematol. Oncol. 2002, 24, 246-249. [CrossRef]

8. Phillips, C.; Sexton, M.; Wheeler, G.; McKenzie, J. Retinoblastoma: Review of 30 years' experience with external beam radiotherapy. Australas. Radiol. 2003, 47, 226-230. [CrossRef]

9. Abramson, D.H.; Beaverson, K.L.; Chang, S.T.; Dunkel, I.J.; McCormick, B. Outcome following initial external beam radiotherapy in patients with Reese-Ellsworth group Vb retinoblastoma. Arch. Ophthalmol. 2004, 122, 1316-1323. [CrossRef]

10. Choi, S.Y.; Kim, M.S.; Yoo, S.; Cho, C.; Ji, Y.; Kim, K.; Seo, Y.; Park, K.D.; Lee, J.; Lee, T.W. Long term follow-up results of external beam radiotherapy as primary treatment for retinoblastoma. J. Korean Med. Sci. 2010, 25, 546-551. [CrossRef] 
11. Camp, D.A.; Dalvin, L.A.; Schwendeman, R.; Lim, L.S.; Shields, C.L. Outcomes of neonatal retinoblastoma in pre-chemotherapy and chemotherapy eras. Indian J. Ophthalmol. 2019, 67, 1997-2004.

12. Beck, M.N.; Balmer, A.; Dessing, C.; Pica, A.; Munier, F. First-line chemotherapy with local treatment can prevent external-beam irradiation and enucleation in low-stage intraocular retinoblastoma. J. Clin. Oncol. 2000, 18, 2881-2887. [CrossRef]

13. Rodriguez-Galindo, C.; Wilson, M.W.; Haik, B.G.; Merchant, T.E.; Billups, C.A.; Shah, N.; Cain, A.; Langston, J.; Lipson, M.; Kun, L.E.; et al. Treatment of intraocular retinoblastoma with vincristine and carboplatin. J. Clin. Oncol. 2003, 21, $2019-2025$. [CrossRef]

14. Gunduz, K.; Gunalp, I.; Yalcindag, N.; Unal, E.; Tacyildiz, N.; Erden, E.; Geyik, P.O. Causes of chemoreduction failure in retinoblastoma and analysis of associated factors leading to eventual treatment with external beam radiotherapy and enucleation. Ophthalmology 2004, 111, 1917-1924. [CrossRef]

15. Schiavetti, A.; Hadjistilianou, T.; Clerico, A.; Bonci, E.; Ragni, G.; Castello, M.A. Conservative therapy in intraocular retinoblastoma: Response/recurrence rate. J. Pediatr. Hematol. Oncol. 2005, 27, 3-6. [CrossRef]

16. Chantada, G.L.; Dunkel, I.J.; Antoneli, C.B.; de Davila, M.T.; Arias, V.; Beaverson, K.; Fandino, A.C.; Chojniak, M.; Abramson, D.H. Risk factors for extraocular relapse following enucleation after failure of chemoreduction in retinoblastoma. Pediatr. Blood Cancer 2007, 49, 256-260. [CrossRef]

17. Lumbroso-Le Rouic, L.; Aerts, I.; Levy-Gabriel, C.; Dendale, R.; Sastre, X.; Esteve, M.; Asselain, B.; Bours, D.; Doz, F.; Desjardins, L. Conservative treatments of intraocular retinoblastoma. Ophthalmology 2008, 115, 1405-1410. [CrossRef]

18. Shin, J.Y.; Kim, J.H.; Yu, Y.S.; Khwarg, S.I.; Choung, H.K.; Shin, H.Y.; Ahn, H.S. Eye-preserving therapy in retinoblastoma: Prolonged primary chemotherapy alone or combined with local therapy. Korean J. Ophthalmol. 2010, 24, 219-224. [CrossRef]

19. Zhao, J.; Dimaras, H.; Massey, C.; Xu, X.; Huang, D.; Li, B.; Chan, H.S.; Gallie, B.L. Pre-enucleation chemotherapy for eyes severely affected by retinoblastoma masks risk of tumor extension and increases death from metastasis. J. Clin. Oncol. 2011, $29,845-851$. [CrossRef]

20. Bartuma, K.; Pal, N.; Kosek, S.; Holm, S.; All-Ericsson, C. A 10-year experience of outcome in chemotherapy-treated hereditary retinoblastoma. Acta Ophthalmol. 2014, 92, 404-411. [CrossRef]

21. Brennan, R.C.; Qaddoumi, I.; Billups, C.A.; Free, T.L.; Haik, B.G.; Rodriguez-Galindo, C.; Wilson, M.W. Comparison of high-risk histopathological features in eyes with primary or secondary enucleation for retinoblastoma. Br. J. Ophthalmol. 2015, 99, 1366-1371. [CrossRef]

22. Berry, J.L.; Kogachi, K.; Aziz, H.A.; McGovern, K.; Zolfaghari, E.; Murphree, A.L.; Jubran, R.; Kim, J.W. Risk of metastasis and orbital recurrence in advanced retinoblastoma eyes treated with systemic chemoreduction versus primary enucleation. Pediatr. Blood Cancer 2017, 64, e26270. [CrossRef]

23. Munier, F.L.; Mosimann, P.; Puccinelli, F.; Gaillard, M.C.; Stathopoulos, C.; Houghton, S.; Bergin, C.; Beck-Popovic, M. First-line intra-arterial versus intravenous chemotherapy in unilateral sporadic group D retinoblastoma: Evidence of better visual outcomes, ocular survival and shorter time to success with intra-arterial delivery from retrospective review of 20 years of treatment. Br. $J$. Ophthalmol. 2017, 101, 1086-1093.

24. Fabian, I.D.; Stacey, A.W.; Chowdhury, T.; Duncan, C.; Karaa, E.K.; Scheimberg, I.; Reddy, M.A.; Sagoo, M.S. High-Risk Histopathology Features in Primary and Secondary Enucleated International Intraocular Retinoblastoma Classification Group D Eyes. Ophthalmology 2017, 124, 851-858. [CrossRef]

25. Shields, C.L.; Bas, Z.; Tadepalli, S.; Dalvin, L.A.; Rao, R.; Schwendeman, R.; Lally, S.E.; Shields, J.A.; Shah, A.; Leahey, A. Long-term (20-year) real-world outcomes of intravenous chemotherapy (chemoreduction) for retinoblastoma in 964 eyes of 554 patients at a single centre. Br. J. Ophthalmol. 2020, 104, 1548-1555. [CrossRef]

26. Gunduz, A.K.; Mirzayev, I.; Temel, E.; Unal, E.; Tacyildiz, N.; Dincaslan, H.; Kose, S.K.; Ozalp Ates, F.S.; Isik, M.U. A 20-year audit of retinoblastoma treatment outcomes. Eye 2020, 34, 1916-1924. [CrossRef]

27. Alkatan, H.M.; Al-Dahmash, S.A.; Almesfer, S.A.; AlQahtani, F.S.; Maktabi, A.M.Y. High-risk features in primary versus secondary enucleated globes with advanced retinoblastoma: A retrospective histopathological study. Int. Ophthalmol. 2020, 40, $2875-2887$. [CrossRef]

28. Abramson, D.H.; Marr, B.P.; Brodie, S.E.; Dunkel, I.; Palioura, S.; Gobin, Y.P. Ophthalmic artery chemosurgery for less advanced intraocular retinoblastoma: Five year review. PLoS ONE 2012, 7, e34120. [CrossRef]

29. Palioura, S.; Gobin, Y.P.; Brodie, S.E.; Marr, B.P.; Dunkel, I.J.; Abramson, D.H. Ophthalmic artery chemosurgery for the management of retinoblastoma in eyes with extensive (>50\%) retinal detachment. Pediatr. Blood Cancer 2012, 59, 859-864. [CrossRef]

30. Thampi, S.; Hetts, S.W.; Cooke, D.L.; Stewart, P.J.; Robbins, E.; Banerjee, A.; Dubois, S.G.; Char, D.; Halbach, V.; Matthay, K. Superselective intra-arterial melphalan therapy for newly diagnosed and refractory retinoblastoma: Results from a single institution. Clin. Ophthalmol. 2013, 7, 981-989. [CrossRef]

31. Shields, C.L.; Kaliki, S.; Al-Dahmash, S.; Rojanaporn, D.; Leahey, A.; Griffin, G.; Jabbour, P.; Shields, J.A. Management of advanced retinoblastoma with intravenous chemotherapy then intra-arterial chemotherapy as alternative to enucleation. Retina 2013, 33, 2103-2109. [CrossRef]

32. Venturi, C.; Bracco, S.; Cerase, A.; Cioni, S.; Galluzzi, P.; Gennari, P.; Vallone, I.M.; Tinturini, R.; Vittori, C.; de Francesco, S.; et al. Superselective ophthalmic artery infusion of melphalan for intraocular retinoblastoma: Preliminary results from 140 treatments. Acta Ophthalmol. 2013, 91, 335-342. [CrossRef] 
33. Bracco, S.; Leonini, S.; De Francesco, S.; Cioni, S.; Gennari, P.; Vallone, I.M.; Piu, P.; Galimberti, D.; Romano, D.G.; Caini, M.; et al. Intra-arterial chemotherapy with melphalan for intraocular retinoblastoma. Br. J. Ophthalmol. 2013, 97, 1219-1221. [CrossRef]

34. Shields, C.L.; Manjandavida, F.P.; Lally, S.E.; Pieretti, G.; Arepalli, S.A.; Caywood, E.H.; Jabbour, P.; Shields, J.A. Intra-arterial chemotherapy for retinoblastoma in 70 eyes: Outcomes based on the international classification of retinoblastoma. Ophthalmology 2014, 121, 1453-1460. [CrossRef] [PubMed]

35. Ong, S.J.; Chao, A.N.; Wong, H.F.; Liou, K.L.; Kao, L.Y. Selective ophthalmic arterial injection of melphalan for intraocular retinoblastoma: A 4-year review. Jpn. J. Ophthalmol. 2015, 59, 109-117. [CrossRef] [PubMed]

36. Yannuzzi, N.A.; Francis, J.H.; Marr, B.P.; Belinsky, I.; Dunkel, I.J.; Gobin, Y.P.; Abramson, D.H. Enucleation vs. Ophthalmic Artery Chemosurgery for Advanced Intraocular Retinoblastoma: A Retrospective Analysis. JAMA Ophthalmol. 2015, 133, 1062-1066. [CrossRef] [PubMed]

37. Akyuz, C.; Kiratli, H.; Sen, H.; Aydin, B.; Tarlan, B.; Varan, A. Intra-Arterial Chemotherapy for Retinoblastoma: A Single-Center Experience. Ophthalmologica 2015, 234, 227-232. [CrossRef]

38. Abramson, D.H.; Daniels, A.B.; Marr, B.P.; Francis, J.H.; Brodie, S.E.; Dunkel, I.J.; Gobin, Y.P. Intra-Arterial Chemotherapy (Ophthalmic Artery Chemosurgery) for Group D Retinoblastoma. PLoS ONE 2016, 11, e0146582. [CrossRef] [PubMed]

39. Abramson, D.H.; Marr, B.P.; Francis, J.H.; Dunkel, I.J.; Fabius, A.W.; Brodie, S.E.; Mondesire-Crump, I.; Gobin, Y.P. Simultaneous Bilateral Ophthalmic Artery Chemosurgery for Bilateral Retinoblastoma (Tandem Therapy). PLoS ONE 2016, 11, e0156806. [CrossRef]

40. Shields, C.L.; Alset, A.E.; Say, E.A.; Caywood, E.; Jabbour, P.; Shields, J.A. Retinoblastoma Control with Primary Intra-arterial Chemotherapy: Outcomes Before and During the Intravitreal Chemotherapy Era. J. Pediatr. Ophthalmol. Strabismus 2016, 53, 275-284. [CrossRef]

41. Chen, M.; Jiang, H.; Zhang, J.; Shen, G.; Jiang, Y.; Li, H.; Liu, Z. Outcome of intra-arterial chemotherapy for retinoblastoma and its influencing factors: A retrospective study. Acta Ophthalmol. 2017, 95, 613-618. [CrossRef]

42. Funes, S.; Sampor, C.; Villasante, F.; Fandino, A.; Manzitti, J.; Sgroi, M.; Neira, P.; Peralta, L.; Lagomarsino, E.; Schaiquevich, P.; et al. Feasibility and results of an intraarterial chemotherapy program for the conservative treatment of retinoblastoma in Argentina. Pediatr. Blood Cancer 2018, 65, e27086. [CrossRef]

43. Ghassemi, F.; Shields, C.L.; Ghadimi, H.; Khodabandeh, A.; Roohipoor, R. Combined intravitreal melphalan and topotecan for refractory or recurrent vitreous seeding from retinoblastoma. JAMA Ophthalmol. 2014, 132, 936-941. [CrossRef]

44. Abramson, D.H.; Fabius, A.W.; Issa, R.; Francis, J.H.; Marr, B.P.; Dunkel, I.J.; Gobin, Y.P. Advanced Unilateral Retinoblastoma: The Impact of Ophthalmic Artery Chemosurgery on Enucleation Rate and Patient Survival at MSKCC. PLoS ONE 2015, 10, e0145436. [CrossRef]

45. Say, E.A.; Iyer, P.G.; Hasanreisoglu, M.; Lally, S.E.; Jabbour, P.; Shields, J.A.; Shields, C.L. Secondary and tertiary intra-arterial chemotherapy for massive persistent or recurrent subretinal retinoblastoma seeds following previous chemotherapy exposure: Long-term tumor control and globe salvage in 30 eyes. J. AAPOS 2016, 20, 337-342. [CrossRef]

46. Shields, C.L.; Mashayekhi, A.; Au, A.K.; Czyz, C.; Leahey, A.; Meadows, A.T.; Shields, J.A. The International Classification of Retinoblastoma predicts chemoreduction success. Ophthalmology 2006, 113, 2276-2280. [CrossRef]

47. Berry, J.L.; Jubran, R.; Kim, J.W.; Wong, K.; Bababeygy, S.R.; Almarzouki, H.; Lee, T.C.; Murphree, A.L. Long-term outcomes of Group D eyes in bilateral retinoblastoma patients treated with chemoreduction and low-dose IMRT salvage. Pediatr. Blood Cancer 2013, 60, 688-693. [CrossRef]

48. Berry, J.L.; Shah, S.; Bechtold, M.; Zolfaghari, E.; Jubran, R.; Kim, J.W. Long-term outcomes of Group D retinoblastoma eyes during the intravitreal melphalan era. Pediatr. Blood Cancer 2017, 64, e26696. [CrossRef]

49. Tuncer, S.; Sencer, S.; Kebudi, R.; Tanyildiz, B.; Cebeci, Z.; Aydin, K. Superselective intra-arterial chemotherapy in the primary management of advanced intra-ocular retinoblastoma: First 4-year experience from a single institution in Turkey. Acta Ophthalmol. 2016, 94, e644-e651. [CrossRef]

50. Shields, C.L.; Bianciotto, C.G.; Jabbour, P.; Ramasubramanian, A.; Lally, S.E.; Griffin, G.C.; Rosenwasser, R.; Shields, J.A. Intraarterial chemotherapy for retinoblastoma: Report No. 1, control of retinal tumors, subretinal seeds, and vitreous seeds. Arch. Ophthalmol. 2011, 129, 1399-1406. [CrossRef]

51. Linn Murphree, A. Intraocular retinoblastoma: The case for a new group classification. Ophthalmol. Clin. N. Am. 2005, 18, 41-53. [CrossRef]

52. European Retinoblastoma Group. Available online: https:/ /www.eurbg.org (accessed on 1 May 2021).

53. Abramson, D.H.; Notterman, R.B.; Ellsworth, R.M.; Kitchin, F.D. Retinoblastoma treated in infants in the first six months of life. Arch. Ophthalmol. 1983, 101, 1362-1366. [CrossRef]

54. Murphree, A.L.; Villablanca, J.G.; Deegan, W.F.; Sato, J.K., 3rd; Malogolowkin, M.; Fisher, A.; Parker, R.; Reed, E.; Gomer, C.J. Chemotherapy plus local treatment in the management of intraocular retinoblastoma. Arch. Ophthalmol. 1996, 114, 1348-1356. [CrossRef]

55. Berry, J.L.; Kogachi, K.; Jubran, R.; Kim, J.W. Loss of fundus view as an indication for secondary enucleation in retinoblastoma. Pediatr. Blood Cancer 2018, 65, e26908. [CrossRef]

56. Bedford, M.A.; Bedotto, C.; Macfaul, P.A. Retinoblastoma. A study of 139 cases. Br. J. Ophthalmol. 1971, 55, 19-27. [CrossRef] 
57. Munier, F.L.; Beck-Popovic, M.; Chantada, G.L.; Cobrinik, D.; Kivela, T.T.; Lohmann, D.; Maeder, P.; Moll, A.C.; Carcaboso, A.M.; Moulin, A.; et al. Conservative management of retinoblastoma: Challenging orthodoxy without compromising the state of metastatic grace. "Alive, with good vision and no comorbidity". Prog. Retin. Eye Res. 2019, 73, 100764. [CrossRef]

58. Abramson, D.H.; Dunkel, I.J.; Brodie, S.E.; Kim, J.W.; Gobin, Y.P. A phase I/II study of direct intraarterial (ophthalmic artery) chemotherapy with melphalan for intraocular retinoblastoma initial results. Ophthalmology 2008, 115, 1398-1404. [CrossRef]

59. Shields, C.L.; Jorge, R.; Say, E.A.T.; Magrath, G.; Alset, A.; Caywood, E.; Leahey, A.M.; Jabbour, P.; Shields, J.A. Unilateral retinoblastoma managed with intravenous chemotherapy versus intra-arterial chemotherapy. Outcomes based on the International Classification of retinoblastoma. Asia Pac. J. Ophthalmol. 2016, 5, 97-103. [CrossRef]

60. Stathopoulos, C.; Gaillard, M.C.; Puccinelli, F.; Maeder, P.; Hadjistilianou, D.; Beck-Popovic, M.; Munier, F.L. Successful conservative treatment of massive choroidal relapse in 2 retinoblastoma patients monitored by ultrasound biomicroscopy and/or spectral domain optic coherence tomography. Ophthalmic Genet. 2018, 39, 242-246. [CrossRef]

61. Abramson, D.H.; Francis, J.H.; Gobin, Y.P. Choroidal Invasion in Retinoblastoma Treated with Intrarterial Chemotherapy. Ophthalmol. Retin. 2018, 2, 9. [CrossRef]

62. Munier, F.L.; Moulin, A.; Gaillard, M.C.; Bongiovanni, M.; Decembrini, S.; Houghton, S.; Beck-Popovic, M.; Stathopoulos, C. Intracameral Chemotherapy for Globe Salvage in Retinoblastoma with Secondary Anterior Chamber Invasion. Ophthalmology 2018, 125, 615-617. [CrossRef]

63. Chhablani, J.; Romanzo, A.; Balmer, A.; Pica, A.; Gaillard, M.C.; Cozza, R.; Moeckli, R.; Munier, F.L. (106)Ruthenium brachytherapy for ciliary recurrence with supraciliary effusion in retinoblastoma. Ophthalmic Genet. 2010, 31, 190-192. [CrossRef]

64. Baroni, L.V.; Sampor, C.; Fandino, A.; Solernou, V.; Demirdjian, G.; de Davila, M.T.; Chantada, G.L. Anterior segment invasion in retinoblastoma: Is it a risk factor for extraocular relapse? J. Pediatr. Hematol. Oncol. 2014, 36, e509-e512. [CrossRef]

65. Chantada, G.; Fandino, A.; Davila, M.T.; Manzitti, J.; Raslawski, E.; Casak, S.; Schvartzman, E. Results of a prospective study for the treatment of retinoblastoma. Cancer 2004, 100, 834-842. [CrossRef]

66. Perez, V.; Sampor, C.; Rey, G.; Parareda-Salles, A.; Kopp, K.; Dabezies, A.P.; Dufort, G.; Zelter, M.; Lopez, J.P.; Urbieta, M.; et al. Treatment of Nonmetastatic Unilateral Retinoblastoma in Children. JAMA Ophthalmol. 2018, 136, 747-752. [CrossRef]

67. Munier, F.L.; Soliman, S.; Moulin, A.P.; Gaillard, M.C.; Balmer, A.; Beck-Popovic, M. Profiling safety of intravitreal injections for retinoblastoma using an anti-reflux procedure and sterilisation of the needle track. Br. J. Ophthalmol. 2012, 96, 1084-1087. [CrossRef] [PubMed]

68. Rao, R.; Honavar, S.G.; Sharma, V.; Reddy, V.A.P. Intravitreal topotecan in the management of refractory and recurrent vitreous seeds in retinoblastoma. Br. J. Ophthalmol. 2018, 102, 490-495. [CrossRef] [PubMed]

69. Francis, J.H.; Schaiquevich, P.; Buitrago, E.; Del Sole, M.J.; Zapata, G.; Croxatto, J.O.; Marr, B.P.; Brodie, S.E.; Berra, A.; Chantada, G.L.; et al. Local and systemic toxicity of intravitreal melphalan for vitreous seeding in retinoblastoma: A preclinical and clinical study. Ophthalmology 2014, 121, 1810-1817. [CrossRef] [PubMed]

70. Berry, J.L.; Bechtold, M.; Shah, S.; Zolfaghari, E.; Reid, M.; Jubran, R.; Kim, J.W. Not All Seeds Are Created Equal: Seed Classification Is Predictive of Outcomes in Retinoblastoma. Ophthalmology 2017, 124, 1817-1825. [CrossRef] [PubMed]

71. Munier, F.L.; Gaillard, M.C.; Balmer, A.; Soliman, S.; Podilsky, G.; Moulin, A.P.; Beck-Popovic, M. Intravitreal chemotherapy for vitreous disease in retinoblastoma revisited: From prohibition to conditional indications. Br. J. Ophthalmol. 2012, 96, 1078-1083. [CrossRef] [PubMed]

72. Shields, C.L.; Douglass, A.M.; Beggache, M.; Say, E.A.; Shields, J.A. Intravitreous Chemotherapy for Active Vitreous Seeding from Retinoblastoma: Outcomes after 192 Consecutive Injections. The 2015 Howard Naquin Lecture. Retina 2016, 36, 1184-1190. [CrossRef]

73. Abramson, D.H.; Marr, B.P.; Dunkel, I.J.; Brodie, S.; Zabor, E.C.; Driscoll, S.J.; Gobin, Y.P. Intra-arterial chemotherapy for retinoblastoma in eyes with vitreous and/or subretinal seeding: 2-year results. Br. J. Ophthalmol. 2012, 96, 499-502. [CrossRef]

74. Stathopoulos, C.; Munier, F.L. Intravitreal chemotherapy. In Clinical Ophthalmic Oncology, 3rd ed.; Retinoblasotma, chapter 15; Springer: Berlin/Heidelberg, Germany, 2019; Volume 6, pp. 179-192.

75. Francis, J.H.; Abramson, D.H.; Gobin, Y.P.; Marr, B.P.; Tendler, I.; Brodie, S.E.; Dunkel, I.J. Efficacy and toxicity of second-course ophthalmic artery chemosurgery for retinoblastoma. Ophthalmology 2015, 122, 1016-1022. [CrossRef] [PubMed]

76. Shields, C.L.; Say, E.A.; Pointdujour-Lim, R.; Cao, C.; Jabbour, P.M.; Shields, J.A. Rescue intra-arterial chemotherapy following retinoblastoma recurrence after initial intra-arterial chemotherapy. J. Français Ophtalmol. 2015, 38, 542-549. [CrossRef] [PubMed]

77. Stathopoulos, C.; Sergenti, J.; Gaillard, M.C.; Munier, F.L.; Daruich, A. Pars plana vitrectomy under melphalan irrigation for recurrent retinal detachment in eyes treated for retinoblastoma: A case report. BMC Ophthalmol. 2020, 20, 34. [CrossRef]

78. Yarovoy, A.A.; Ushakova, T.L.; Gorshkov, I.M.; Polyakov, V.G.; Golubeva, O.V.; Gorovtsova, O.V.; Krivovyaz, O.S. Intraocular surgery with melphalan irrigation for vitreous hemorrhage in an only eye with retinoblastoma. Eur. J. Ophthalmol. 2015, 26, e17-e19. [CrossRef] [PubMed]

79. Saumya Pal, S.; Gopal, L.; Khetan, V.; Nagpal, A.; Sharma, T. Rhegmatogenous retinal detachment following treatment for retinoblastoma. J. Pediatr. Ophthalmol. Strabismus 2010, 47, 349-355. [CrossRef]

80. Stathopoulos, C.; Gaillard, M.C.; Moulin, A.; Puccinelli, F.; Beck-Popovic, M.; Munier, F.L. Intravitreal Anti-Vascular Endothelial Growth Factor for the Management of Neovascularization in Retinoblastoma after Intravenous and/or Intraarterial Chemotherapy: Long-Term Outcomes in a Series of 35 Eyes. Retina 2019, 39, 2273-2282. [CrossRef] 
81. Munier, F.L.; Gaillard, M.-C.; Decembrini, S.; Beck-Popovic, M. Aqueous seeding: Fall of the ultimate intraocular retinoblastoma sanctuary by a new in situ chemotherapy technique. Investig. Ophthalmol. Vis. Sci. 2015, 56, 1663.

82. Munier, F.L.; Gaillard, M.C.; Decembrini, S.; Bongiovanni, M.; Beck-Popovic, M. Intracameral Chemotherapy (Melphalan) for Aqueous Seeding in Retinoblastoma: Bicameral Injection Technique and Related Toxicity in a Pilot Case Study. Ocul. Oncol. Pathol. 2017, 3, 149-155. [CrossRef]

83. Cassoux, N.; Aerts, I.; Lumbroso-Le Rouic, L.; Freneaux, P.; Desjardins, L. Eye Salvage with Combination of Intravitreal and Intracameral Melphalan Injection for Recurrent Retinoblastoma with Anterior Chamber Involvement: Report of a Case. Ocul. Oncol. Pathol. 2017, 3, 129-132. [CrossRef] [PubMed]

84. Paez-Escamilla, M.; Bagheri, N.; Teira, L.E.; Corrales-Medina, F.F.; Harbour, W. Intracameral Topotecan Hydrochloride for Anterior Chamber Seeding of Retinoblastoma. JAMA Ophthalmol. 2017, 135, 1453-1454. [CrossRef] [PubMed]

85. Smith, S.J.; Smith, B.D. Evaluating the risk of extraocular tumour spread following intravitreal injection therapy for retinoblastoma: A systematic review. Br. J. Ophthalmol. 2013, 97, 1231-1236. [CrossRef] [PubMed]

86. Wyse, E.; Handa, J.T.; Friedman, A.D.; Pearl, M.S. A review of the literature for intra-arterial chemotherapy used to treat retinoblastoma. Pediatr. Radiol. 2016, 46, 1223-1233. [CrossRef] [PubMed]

87. Yousef, Y.A.; Soliman, S.E.; Astudillo, P.P.; Durairaj, P.; Dimaras, H.; Chan, H.S.; Heon, E.; Gallie, B.L.; Shaikh, F. Intra-arterial Chemotherapy for Retinoblastoma: A Systematic Review. JAMA Ophthalmol. 2016, 134, 584-591. [CrossRef]

88. Levin, M.H.; Gombos, D.S.; O’Brien, J.M. Intra-arterial chemotherapy for advanced retinoblastoma: Is the time right for a prospective clinical trial? Arch. Ophthalmol. 2011, 129, 1487-1489. [CrossRef]

89. Abramson, D.H.; Shields, C.L.; Jabbour, P.; Teixeira, L.F.; Fonseca, J.R.F.; Marques, M.C.P.; Munier, F.L.; Puccinelli, F.; Hadjistilianou, T.; Bracco, S.; et al. Metastatic deaths in retinoblastoma patients treated with intraarterial chemotherapy (ophthalmic artery chemosurgery) worldwide. Int. J. Retin. Vitr. 2017, 3, 40. [CrossRef] [PubMed]

90. Fabian, I.D.; Puccinelli, F.; Gaillard, M.C.; Beck-Popovic, M.; Munier, F.L. Diagnosis and management of secondary epipapillary retinoblastoma. Br. J. Ophthalmol. 2017, 101, 1412-1418. [CrossRef]

91. Stathopoulos, C. OCT imaging of Schlemm's canal invasion in a retinoblastoma patient. Ophthalmology 2021, 128, 876. [CrossRef] [PubMed]

92. Shields, J.A.; Sanborn, G.E.; Augsburger, J.J.; Orlock, D.; Donoso, L.A. Fluorescein angiography of retinoblastoma. Trans. Am. Ophthalmol. Soc. 1982, 80, 98-112.

93. Li, Z.; Guo, J.; Xu, X.; Wang, Y.; Mukherji, S.K.; Xian, J. Diagnosis of Postlaminar Optic Nerve Invasion in Retinoblastoma with MRI Features. J. Magn. Reson. Imaging 2020, 51, 1045-1052. [CrossRef] [PubMed]

94. Cho, S.J.; Kim, J.H.; Baik, S.H.; Sunwoo, L.; Bae, Y.J.; Choi, B.S. Diagnostic performance of MRI of post-laminar optic nerve invasion detection in retinoblastoma: A systematic review and meta-analysis. Neuroradiology 2021, 63, 499-509. [CrossRef]

95. Dittner-Moormann, S.; Reschke, M.; Abbink, F.C.H.; Aerts, I.; Atalay, H.T.; Fedorovna Bobrova, N.; Biewald, E.; Brecht, I.B.; Caspi, S.; Cassoux, N.; et al. Adjuvant therapy of histopathological risk factors of retinoblastoma in Europe: A survey by the European Retinoblastoma Group (EURbG). Pediatr. Blood Cancer 2021, 68, e28963. [CrossRef] [PubMed]

96. Sreelakshmi, K.V.; Chandra, A.; Krishnakumar, S.; Natarajan, V.; Khetan, V. Anterior Chamber Invasion in Retinoblastoma: Not an Indication for Adjuvant Chemotherapy. Investig. Opthalmol. Vis. Sci. 2017, 58, 4654-4661. [CrossRef] [PubMed]

97. Suryawanshi, P.; Ramadwar, M.; Dikshit, R.; Kane, S.V.; Kurkure, P.; Banavali, S.; Viswanathan, S. A study of pathologic risk factors in postchemoreduced, enucleated specimens of advanced retinoblastomas in a developing country. Arch. Pathol. Lab. Med. 2011, 135, 1017-1023. [CrossRef] [PubMed]

98. Berry, J.L.; Munier, F.L.; Gallie, B.L.; Polski, A.; Shah, S.; Shields, C.L.; Gombos, D.S.; Ruchalski, K.; Stathopoulos, C.; Shah, R.; et al. Response criteria for intraocular retinoblastoma: RB-RECIST. Pediatr. Blood Cancer 2021, 68, e28964. [CrossRef]

99. Berry, J.L.; Xu, L.; Murphree, A.L.; Krishnan, S.; Stachelek, K.; Zolfaghari, E.; McGovern, K.; Lee, T.C.; Carlsson, A.; Kuhn, P.; et al. Potential of Aqueous Humor as a Surrogate Tumor Biopsy for Retinoblastoma. JAMA Ophthalmol. 2017, 135, 1221-1230. [CrossRef] 